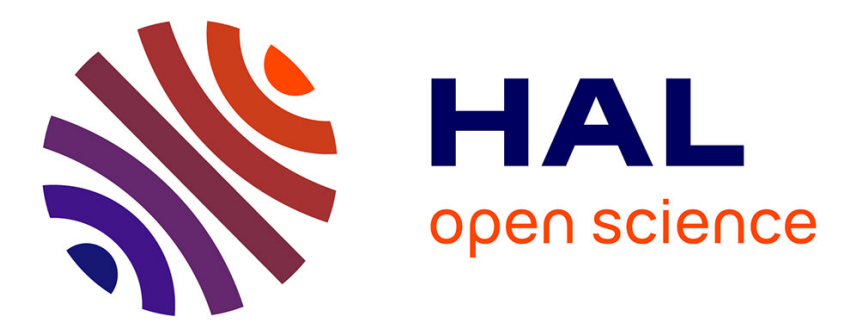

\title{
Development and assessment of a coupled strategy for conjugate heat transfer with Large Eddy Simulation: Application to a cooled turbine blade
}

Florent Duchaine, Alban Corpron, Lorenzo Pons, Vincent Moureau, Franck

Nicoud, Thierry Poinsot

\section{To cite this version:}

Florent Duchaine, Alban Corpron, Lorenzo Pons, Vincent Moureau, Franck Nicoud, et al.. Development and assessment of a coupled strategy for conjugate heat transfer with Large Eddy Simulation: Application to a cooled turbine blade. International Journal of Heat and Fluid Flow, 2009, 30 (6), pp.1129-1141. 10.1016/j.ijheatfluidflow.2009.07.004 . hal-00803384

\section{HAL Id: hal-00803384 https://hal.science/hal-00803384}

Submitted on 22 Mar 2013

HAL is a multi-disciplinary open access archive for the deposit and dissemination of scientific research documents, whether they are published or not. The documents may come from teaching and research institutions in France or abroad, or from public or private research centers.
L'archive ouverte pluridisciplinaire HAL, est destinée au dépôt et à la diffusion de documents scientifiques de niveau recherche, publiés ou non, émanant des établissements d'enseignement et de recherche français ou étrangers, des laboratoires publics ou privés. 


\title{
Development and assessment of a coupled strategy for conjugate heat transfer with Large Eddy Simulation. Application to a cooled turbine blade
}

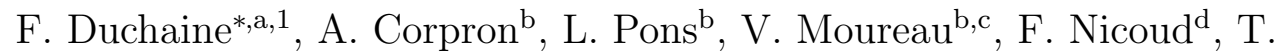 \\ Poinsot ${ }^{\mathrm{e}}$ \\ ${ }^{a}$ CERFACS, 42 av. Coriolis, 31057 Toulouse Cedex 01, France \\ ${ }^{b}$ Turbomeca (Safran Group), Bordes, France \\ ${ }^{c}$ Coria, CNRS, St Etienne du Rouvray, France \\ ${ }^{d}$ University Montpellier II, Montpellier, France \\ ${ }^{e}$ IMFT, CNRS, Toulouse, France
}

\section{Abstract}

Although Large Eddy Simulation (LES) is identified today as the most promising method for turbulent flow problems, few applications of LES coupled to heat transfer solvers in solids have been published. This paper describes a coupling strategy of a LES solver and a heat transfer code within solids on parallel architectures. The numerical methods used in both solvers are briefly recalled before discussing the coupling strategy in terms of physical quantities to exchange (fluxes and temperatures), stability and parallel efficiency. The stability study is performed using an amplification matrix analysis on a one-dimensional case and allows the determination and optimization of coupling parameters. The coupled tool is then applied to a cooled turbine blade model where results demonstrate both the efficiency of the parallel implementation and the quality of the results. Coupled and

\footnotetext{
*Corresponding author. Tel.: (33) (0)5 611931 22; fax: (33) (0)5 61193000

Email address: florent.duchaine@cerfacs.fr (F. Duchaine)
} 
non-coupled simulations are compared to experimental results and discussed in terms of cooling efficiency and flow structures.

Key words: Conjugate Heat Transfer, Large Eddy Simulation, Code

Coupling, Stability Analysis 


\section{Introduction}

Determination of heat loads, as wall temperatures and heat fluxes, is a key issue in combustion (Lakshminarayana, 1996; Lefebvre, 1999; Schiele and Wittig, 2000; Dunn, 2001; Bunker, 2007): the interaction of hot gases and reacting flows with colder walls is an important phenomenon in combustion chambers and a main design constraint in gas turbines. After combustion, the interaction of the hot burnt gases with the high pressure stator and the first turbine blades conditions the temperature and pressure levels reached in

the combustor, and therefore the engine efficiency. Numerical simulations of the thermal interaction between fluid flows and solids offer new design paths to diminish development costs through important reductions of the number of experimental tests.

When a Computational Fluid Dynamics (CFD) solver is coupled to a heat transfer solver, the accuracy of the coupled tool is generally controlled by the fluid code. Conventional CFD techniques use Reynolds Averaged NavierStokes (RANS) methods. Such approaches cannot capture all complex effects in turbomachinery flows and looking for more precise flow solvers is a usual objective in this field. Direct Numerical Simulation (DNS) methods cannot offer such an alternative solution because of their computational cost. Recent progresses in Large Eddy Simulation (LES) (Sagaut, 2000; Mahesh et al., 2004; Poinsot and Veynante, 2005) and the continuously increasing computer power offered by the newly developed parallel computer architectures, allow to accurately predict turbulent flows in complex geometries (Acharya et al., 2001; Azzi and Lakehal, 2002; Rozati, 2007; Boudier et al., 2007). As LES is still a computationally expensive method, the aim of this paper is to develop 
and assess a suitable strategy based on LES to efficiently converge to steady thermal states.

There are two basic approaches to solve Conjugate Heat Transfer (CHT) problems. The first one is a direct coupling where the different fields are solved simultaneously in a large system of equations by a monolithic solver (Kao and Liou, 1997; Han et al., 2001; Rahman et al., 2005; Luo and Razinsky, 2007; Ganesan, 2007). The second approach consists in solving each set of field equations separately with dedicated solvers that exchange boundary conditions (Heselhaus and Vogel, 1995; Sondak and Dorney, 2000; Papanicolaou et al., 2001; Garg, 2002; Bohn et al., 2005). This solution has the advantage of using existing state-of-the-art codes to solve fluid and solid equations and of being able to exchange one solver with another easily (Alonso et al., 2006). The main drawback of this coupling methodology is that an adapted CHT framework is requested for the simulations especially on parallel machines. The performances of such a coupling framework are linked to (1) the strategy to couple the solvers in an accurate and stable fashion as well as (2) the exchange of information between the solvers in an efficient and scalable fashion when using a large number of processors (Alonso et al., 2006).

This paper investigates important issues for fully parallel and coupled CHT based on LES for flow models:

- The boundary conditions applied to the fluid and solid codes, including the variables shared by the codes, are critical for the accuracy and stability of the computations,

- In gas turbine applications, the time scales of the flow and of the solid are generally very different. As a consequence, the frequency of ex- 
changes between the codes and the synchronization of the solvers influence the stability as well as the restitution time of the computations.

The document is organized as follows. The fluid and solid solvers are presented in section 1. Section 2 is dedicated to the CHT framework. The coupling methodology (boundary conditions and synchronization of the solvers) is described in sub-section 2.1. The stability study (sub-section 2.2) is performed on a one-dimensional problem using an amplification matrix analysis. The strategy is validated (section 3) through comparisons of pressure and temperature fields on the experimental film-cooled blade. After a short description of the configuration (sub-section 3.1) and of solid and fluid discretizations (sub-section 3.2), an adiabatic LES is discussed (subsection 3.3). Thermal results given by the CHT methodology are presented in sub-section 3.4 which also describes the main flow structures controlling heat transfer. Finally, sub-section 3.5 proposes efficiency analyses of the coupled strategy.

\section{Numerical approach}

\subsection{Governing equations for flow LES models.}

LES of reacting flows involves the spatial Favre filtering operation that reduces for spatially, temporally invariant and localised filter functions (Vreman et al., 1994) to:

$$
\widetilde{f(\mathbf{x}, t)}=\frac{1}{\overline{\rho(\mathbf{x}, t)}} \int_{-\infty}^{+\infty} \rho\left(\mathbf{x}^{\prime}, t\right) f\left(\mathbf{x}^{\prime}, t\right) G\left(\mathbf{x}^{\prime}-\mathbf{x}\right) d \mathbf{x}^{\prime}
$$

where $G$ denotes the filter function. 
In the mathematical description of compressible turbulent flows the primary variables are the volumic mass fraction $\rho(\mathbf{x}, t)$, the velocity vector $u_{i}(\mathbf{x}, t)$ and the total energy $E(\mathbf{x}, t) \equiv e_{s}+1 / 2 u_{i} u_{i}$.

The fluid follows the ideal gas law, $p=\rho r T$ and $e_{s}=\int_{0}^{T} C_{p} d T-p / \rho$, where $e_{s}$ is the sensible energy, $T$ the temperature, $C_{p}$ the fluid heat capacity at constant pressure and $r$ is the mixture gas constant. The LES solver takes into account changes of heat capacity with temperature using tabulated values of heat capacities. The viscous stress tensor and the heat diffusion vector use classical gradient approaches. The fluid viscosity follows Sutherland's law and the heat diffusion coefficient follows Fourier's law. The application of the filtering operation to the instantaneous set of compressible Navier-Stokes transport equations yields the LES transport equations (Poinsot and Veynante, 2005) which contain so-called Sub-Grid Scale (SGS) quantities that need modelling (Sagaut, 2000; Ferziger, 1977). The unresolved SGS stress tensor $\overline{\tau_{i j}}$ is modelled using the Boussinesq assumption (Smagorinsky, 1963; Pope, 2000; Chassaing, 2000):

$$
{\overline{\tau_{i j}}}^{t}-\frac{1}{3}{\overline{\tau_{k k}}}^{t} \delta_{i j}=-2 \bar{\rho} \nu_{t} \widetilde{S}_{i j} \text { with } \widetilde{S}_{i j}=\frac{1}{2}\left(\frac{\partial \widetilde{u}_{i}}{\partial x_{j}}+\frac{\partial \widetilde{u}_{j}}{\partial x_{i}}\right)-\frac{1}{3} \frac{\partial \widetilde{u}_{k}}{\partial x_{k}} \delta_{i j}
$$

In Eq. (2), $\widetilde{S}_{i j}$ is the resolved strain rate tensor and $\nu_{t}$ is the SGS turbulent viscosity. The Wall Adapting Linear Eddy (WALE) model (Nicoud and Ducros, 1999) is chosen to model the SGS viscosity:

$$
\nu_{t}=\left(C_{w} \Delta\right)^{2} \frac{\left(s_{i j}^{d} s_{i j}^{d}\right)^{3 / 2}}{\left(\widetilde{S}_{i j} \widetilde{S}_{i j}\right)^{5 / 2}+\left(s_{i j}^{d} s_{i j}^{d}\right)^{5 / 4}} \text { with } s_{i j}^{d}=\frac{1}{2}\left({\widetilde{g_{i j}}}^{2}+{\widetilde{g_{j i}}}^{2}\right)+\frac{1}{3}{\widetilde{g_{k k}}}^{2} \delta_{i j}
$$

In Eq. (3), $\Delta$ denotes the filter characteristic length (approximated by the cubic-root of the cell volume), $C_{w}$ is a model constant equal to 0.5 and $\widetilde{g_{i j}}$ is the resolved velocity gradient. 
The SGS energy flux ${\overline{q_{i}}}^{t}$ is modelled using a SGS turbulent heat conductivity obtained from $\nu_{t}$ by $\lambda_{t}=\bar{\rho} \nu_{t} C_{p} / P r_{t}$ where $P r_{t}=0.7$ is a constant turbulent Prandtl number:

$$
{\overline{q_{i}}}^{t}=-\lambda_{t} \frac{\partial \widetilde{T}}{\partial x_{i}}
$$

In Eq. (4), $\widetilde{T}$ is the Favre filtered temperature which satisfies the modified filtered state equation $\bar{p}=\bar{\rho} r \widetilde{T}$ (Moin et al., 1991; Erlebacher et al., 1992; Ducros et al., 1996; Comte, 1996). Although the performances of the closures could be improved through the use of a dynamic formulation (Moin et al., 1991; Lilly, 1992; Germano, 1992; Ghosal and Moin, 1995; Meneveau et al., 1996) Eq. 2-4 are considered sufficient to address the present preliminary investigation.

The WALE subgrid model is used in conjunction with no-slip wall conditions. This model is designed to provide correct levels of turbulent viscosity down to the wall and no wall model is required. Total pressure and total temperature with velocity angle are imposed using the Navier-Stokes Characteristic Boundary Condition (NSCBC) formalism (Poinsot and Lele, 1992) at the inlets of the fluid domain. Static pressures are enforced at outlet boundaries in characteristic NSCBC form.

\subsection{Governing equations for solid heat transfer models.}

Heat transfer in solid domains is described by the energy conservation:

$$
\rho_{s} C_{s} \frac{\partial T(\mathbf{x}, t)}{\partial t}=-\frac{\partial q_{i}}{\partial x_{i}}
$$


where $T$ is the temperature, $\rho_{s}$ the density, $C_{s}$ is the heat capacity and $q$ the conduction heat flux. The heat diffusion follows Fourier's law:

$$
q_{i}=-\lambda_{s} \frac{\partial T}{\partial x_{i}}
$$

where $\lambda_{s}$ is the heat conductivity of the medium. The solid solver takes into account local changes of heat capacity and conductivity with temperature.

\subsection{Numerical schemes.}

The parallel LES code (Schönfeld and Poinsot, 1999; Moureau et al., 2005; Mendez and Nicoud, 2008; Roux et al., 2008) solves the full compressible Navier-Stokes equations using a cell-vertex/finite element approximation and Taylor-Galerkin weighted residual central distribution scheme (Donea and Huerta, 2003). This explicit scheme, which provides third-order accuracy on hybrid meshes, is particularly adequate for low-dissipation requirements of LES applications (Colin and Rudgyard, 2000). Boundary conditions are handled with the NSCBC formulation (Poinsot and Veynante, 2005; Moureau et al., 2005).

The parallel conduction solver is based on the same data structure and uses an explicit scheme for time advancement.

\section{Conjugate heat transfer}

During this work, only a thermal steady state solution within the blade structure was sought for. Since the two solvers are explicit and time dependent, the coupling strategy between the solvers requires a specific approach discussed in sub-section 2.1. The stability of the approach is studied in subsection 2.2 by determining stability limits in a simplified $1 \mathrm{D}$ case. 


\subsection{Coupling strategies}

The resolution of CHT problems on massively parallel machines involves two main issues:

- The boundary conditions applied to the fluid and solid codes control the precision and stability of the computations (sub-section 2.1.1).

- The frequency of exchanges between the codes and the synchronization of the solvers influence the stability as well as the restitution time of the computations (sub-section 2.1.2).

\subsubsection{Boundary conditions}

At a fluid/solid interface $\Gamma_{f s}$, heat flux and temperature are continuous:

$$
\left\{\begin{array}{l}
\phi_{f}^{I}=\phi_{s}^{I} \\
T_{f}^{I}=T_{s}^{I}
\end{array}, \quad I \in \Gamma_{f s}\right.
$$

with $\left(\phi_{f}^{I}, \phi_{s}^{I}\right)$ the fluid and solid heat fluxes at the interface point $I$ and $\left(T_{f}^{I}, T_{s}^{I}\right)$ the fluid and solid temperatures at the same location. Thus, natural variables to share between the codes are $\phi$ and $T$ at the interfaces. However, it is usually not efficient to directly impose $\phi$ or $T$ on either one of the solver. Typical strategies to treat CHT problems (Sondak and Dorney, 2000; Bohn et al., 2005) consist in choosing an adequate composition of variables $\phi_{s}^{I}$ and $T_{s}^{I}$ coming from the solid computation as boundary conditions for the fluid domain. The flow solution is computed with this set of boundary conditions during $n_{f}$ iterations of the fluid solver. Similarly, an adequate composition of variables $\phi_{f}^{I}$ and $T_{f}^{I}$ coming from the fluid computation is imposed as boundary conditions for the solid domain during $n_{s}$ iterations of the thermal code. 
To reach a thermal steady state, these steps are repeated until convergence of boundary variables $\phi^{I}$ and $T^{I}$. The solution retained here is to write the continuity of heat flux and temperature across the interface $\Gamma_{f s}$ (Eq. 7) in the following form (Chemin, 2006):

$$
\left\{\begin{array}{ccc}
T_{f}^{I} & = & T_{s}^{I} \\
\phi_{f}^{I}+h T_{f}^{I} & = & \phi_{s}^{I}+h T_{s}^{I}
\end{array}\right.
$$

where $h$ is a positive numerical coupling relaxation parameter (CRP) which has the dimensions of a convection coefficient. As recommended by previous studies to improve the stability of the coupled scheme (Giles, 1997; Chemin, 2006; Radenac, 2006; Roe et al., 2008), the temperature from the solid is imposed to the fluid domain with a Dirichlet condition:

$$
T_{f}^{I, n}=T_{s}^{I}, \text { with } n=1, n_{f}
$$

The resulting mixed boundary condition for the structure is:

$$
\phi_{s}^{I, n}=\phi_{f}^{I}+h\left(T_{f}^{I}-T_{s}^{I, n}\right), \text { with } n=1, n_{s}
$$

Both $\phi_{s}^{I, n}$ and $T_{s}^{I, n}$ of the mixed formulation (Eq. 10) converge to steady state between two successive updates of $\phi_{f}^{I}$ and $T_{f}^{I}$. The stability of the mixed condition for the solid domain used in conjunction with Dirichlet boundary temperature for the fluid depends on the value of the CRP $h$ and on update frequency. Sub-section 2.1.2 gives arguments concerning update frequencies adopted within this study and sub-section 2.2 proposes a stability analysis of the coupled strategy based on a simplified configuration.

\subsubsection{Synchronization of the solvers}

In a gas turbine, a blade submitted to the hot stream exiting from a combustion chamber has a characteristic time scale $\tau_{s}$, based on a reference 
length and thermal diffusivity, of the order of a few seconds, while the flowthrough time along the blade $\tau_{f}$ is less than $1 \mathrm{~ms}$. Hence, the convergence to a thermal steady state of the conjugate system is controlled by the solid.

Let us consider that between two updates of the boundary values, the flow is advanced in time of a quantity $\alpha_{f} \tau_{f}$ and the solid is advanced of a time $\alpha_{s} \tau_{s}$ where $\alpha_{f}$ and $\alpha_{s}$ are two non-dimensional constants. Then, two limit cases are of interest: (1) $\alpha_{s}=\alpha_{f}$ ensures that both solid and fluid converge to steady state at the same rate and (2) $\alpha_{f} \tau_{f}=\alpha_{s} \tau_{s}$ ensures that the two solvers are synchronized in physical time. This last case is needed for fully coupled unsteady computation. As for the present application only the steady state temperature field in the solid is requested, case (1) $\alpha=\alpha_{s}=\alpha_{f}$ is used. In the following, $\alpha$ is called the coupling synchronization time parameter (CSTP). The numbers of iterations of the fluid and solid solver, respectively $n_{f}$ and $n_{s}$, are linked to fluid and solid time steps $\Delta t_{f}$ and $\Delta t_{s}$ by:

$$
\begin{aligned}
& n_{f}=\alpha \tau_{f} / \Delta t_{f} \\
& n_{s}=\alpha \tau_{s} / \Delta t_{s}
\end{aligned}
$$

On a parallel machine, codes for the fluid and for the structure may be run together or sequentially. Figure 1 shows how heat fluxes and temperature are exchanged in a mode called Sequential Coupling Strategy (SCS) (Duchaine et al., 2008) or staggered solution procedure (Felippa et al., 2001): at synchronization $n_{c p l}$ of the solvers (the subscript $c p l$ refer to CouPLing quantities), after a physical time $\alpha \tau_{f}$ the fluid solver provides fluxes and temperatures to the solid solver which then starts and gives back temperatures (physical duration $\alpha \tau_{s}$ ). In SCS, the codes are loaded into the parallel machine sequentially and each solver uses all available processors $(P)$. Most exist- 
ing applications that use SCS are based on steady state codes (i.e. RANS solvers for the fluid) (Divo et al., 2002; Heidmann et al., 2003; Mercier et al., 2006; Verstraete et al., 2007). Another solution is Parallel Coupling Strategy (PCS) (Duchaine et al., 2008), or parallel staggered procedure (Felippa et al., 2001), where both solvers run together using informations obtained from the other solver at the previous coupling iteration (Fig. 2). PCS is employed to study transient or unsteady phenomena (Montenay et al., 2000; Hegab et al., 2001; dos Santos et al., 2008; Duchaine et al., 2008). In the case of PCS, the two solvers must share the $P=P_{s}+P_{f}$ processors. To first order, ensuring load balancing requires that the $P_{s}$ and $P_{f}$ processors dedicated to the solid and the fluid, respectively, must be such that:

$$
\frac{P_{f}}{P}=\frac{1}{1+\mathcal{T}_{s} / \mathcal{T}_{f}}
$$

where $\mathcal{T}_{s}$ and $\mathcal{T}_{f}$ are the execution times of the solid and fluid solvers on one processor to compute physical times $\alpha \tau_{s}$ and $\alpha \tau_{f}$ respectively. Perfect scaling for both solvers is assumed in Eq. 12. Efficient implementation of PCS requires a software to manage the parallel execution of the solvers as well as the data exchanges during their execution. In order to insure the performance of the PCS, a code coupler, initially developed for ocean-atmosphere coupling (Lagarde et al., 2001; Buis et al., 2005), has been used here.

Both SCS and PCS have been tested: to deal with a fully non stationary coupled problem with synchronization in physical time $\left(\alpha_{f} \tau_{f}=\alpha_{s} \tau_{s}\right)$, PCS is recommended (Hegab et al., 2001). However, when studying the convergence to a steady thermal state where $\alpha=\alpha_{f}=\alpha_{s}$, the choice of the method is open and will be addressed in sub-section 3.5. 


\subsection{Stability of the coupling methodology}

The coupling methodology used to reach a steady thermal state is composed of the following set of boundary conditions:

$$
\left\{\begin{array}{lll}
\text { Fluid (Dirichlet): } & T_{f}^{I, i_{f}}=T_{s}^{I}, & i_{f}=1, n_{f} \\
\text { Solid (mixed): } & \phi_{s}^{I, i_{s}}=\phi_{f}^{I}+h\left(T_{f}^{I}-T_{s}^{I, i_{s}}\right), & i_{s}=1, n_{s}
\end{array}\right.
$$

Boundary conditions of Eq. 13 are applied to fluid and solid domains during the $n_{f}$ and $n_{s}$ iterations of the solvers between two consecutive updates. In Eq. 13, the coupling relaxation parameter $h$ and the coupling synchronization time parameter $\alpha$ are adjustable. The other free parameter is the mode of synchronization: SCS or PCS. Before applying the CHT methodology to a full configuration, it is interesting to study its stability in terms of $h$ and $\alpha$. This is done here in a simplified 1D case with the PCS.

\subsubsection{Description of the simplified conjugate heat transfer problem}

In coupled simulations, modes which are normal to the interface between the fluid and the solid can become unstable. Hence, the stability of the PCS with Dirichlet / mixed set of boundary conditions is analyzed here with a 1D purely thermal diffusion problem $\mathcal{P}_{\mathcal{S C H} \mathcal{T}}$ (Simplified conjugate heat transfer problem). As mentioned by Giles (Giles, 1997), since there is no velocity component normal to the solid boundaries, it is appropriate in a $1 \mathrm{D}$ model to omit convection terms. Stability for problem $\mathcal{P}_{\mathcal{S C H} \mathcal{T}}$ is a necessary condition for real applications but may not be sufficient. Nevertheless, understanding 1D instabilities clearly gives insight into the potential instabilities in 2D and 3D complex computations.

The problem $\mathcal{P}_{\mathcal{S C H} \mathcal{T}}$ is a system composed of two domains with constant properties. Domain \#1 has the thermal properties (thermal conductivity, 
heat capacity and density) of plexiglass (Tab. 1) while domain \#2 has the thermal properties of air at $328 \mathrm{~K}$ (Tab. 2). The interface between the domains at $x=0$ is noted $\Gamma$. Dirichlet conditions on temperature are imposed at $x=b_{1}\left(b_{1}<0\right)$ and $x=b_{2}\left(b_{2}>0\right)$. To solve the problem $\mathcal{P}_{\mathcal{S C H T}}$, the heat diffusion equation is considered in both domains:

$$
\rho_{i} C_{i} \frac{\partial T_{i}(x)}{\partial t}=\lambda_{i} \frac{\partial^{2} T_{i}(x)}{\partial x^{2}}, i=1,2
$$

with the boundary and continuity conditions:

$$
\left\{\begin{array}{l}
T_{1}\left(x=b_{1}\right)=T_{b_{1}} \\
T_{2}\left(x=b_{2}\right)=T_{b_{2}} \\
\phi_{1}(x=0)=\phi_{2}(x=0) \\
T_{1}(x=0)=T_{2}(x=0)
\end{array}\right.
$$

For the sake of simplicity, Eq. 14 is discretized using a forward Euler time differencing and a classical second-order central difference scheme on uniform grids in each domain:

$$
T_{i, j}^{n+1}=T_{i, j}^{n}+\mathcal{F}_{i}\left(T_{i, j+1}^{n}-2 T_{i, j}^{n}+T_{i, j-1}^{n}\right), i=1,2
$$

where $\mathcal{F}_{i}=\frac{\lambda_{i} \Delta t_{i}}{\rho_{i} C_{i} \Delta x_{i}^{2}}, \Delta t_{i}$ and $\Delta x_{i}$ are the Fourier number, the time step and the mesh size of domain $i$ respectively. For a periodic domain with uniform thermo-physical properties, the stability of the discretization given by Eq. 16 is ensured when $\mathcal{F}_{i} \leq 0.5$ (Burnett, 1987). In Eq. 16, $T_{i, j}^{n}$ is given by:

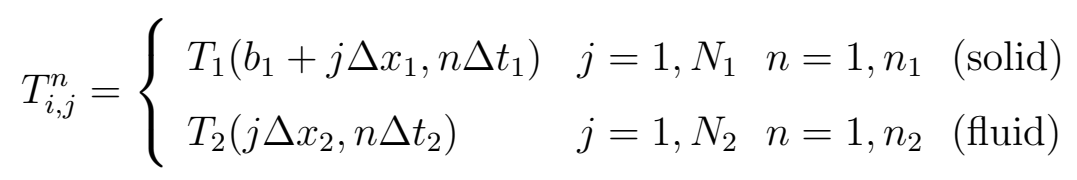

where $N_{1}$ and $N_{2}$ are the number of mesh points of domain \#1 and \#2 respectively. Previous works dealing with numerical stability of coupled thermal 
computations (Giles, 1997; Chemin, 2006; Radenac, 2006; Roe et al., 2008) were done with the very restrictive conditions $n_{1}=n_{2}=1$ and $\Delta t_{1}=\Delta t_{2}$. In this study, the coupled solvers conserve their own time step based on stand-alone numerical stability. Moreover, the solvers can integrate Eq. 16 with different number iterations $n_{1}$ and $n_{2}$ between two updates. To mimic the conditions of the blade heat transfer problem, the spatial and time discretizations $\Delta x_{i}$ and $\Delta t_{i}$ are similar to those of the computation with heat conduction solver for domain \#1 and the LES code for domain \#2.

The discretized heat flux used for boundary conditions are approximated with first-order differences. After the synchronization number $n_{c p l}$ in PCS (Fig. 2), the boundary conditions applied to domain \#1 and \#2 during $n_{1}$ and $n_{2}$ iterations respectively are:

$$
\left\{\begin{array}{l}
T_{1,1}^{i_{1}, n_{c p l}}=T_{b_{1}} \\
\phi_{1, N_{1}}^{i_{1}, n_{c p l}}=\bar{\phi}_{2,1}^{n_{c p l}-1}+h\left(T_{2,1}^{n_{2}, n_{c p l}-1}-T_{1, N_{1}}^{i_{1}, n_{c p l}}\right) \\
T_{2,1}^{i_{2}, n_{c p l}}=T_{1, N_{1}}^{n_{1}, n_{c p l}-1} \\
T_{2,1}^{i_{2}, n_{c p l}}=T_{b_{2}} \\
\text { with } i_{1}=1, n_{1} \text { and } i_{2}=1, n_{2}
\end{array}\right.
$$

where $\bar{\phi}_{2,1}^{n_{c p l}-1}$ is the mean value of the heat flux $\phi_{2,1}^{i_{2}, n_{c p l}-1}$ at the interface in domain \#2 over the $n_{2}$ iterations. This mean heat flux is introduced in the mixed condition due to the use of a LES solver, intrinsically non stationary, for target applications.

\subsubsection{Stability of the solid discretization}

Before studying the coupled problem $\mathcal{P}_{\mathcal{S C H} \mathcal{T}}$, let us consider only domain \#1 to analyze the effect of the mixed boundary condition (Eq. 13) on stability. 
Domain \#1 is discretized with Eq. 16. The mixed boundary condition is applied at $x=0$ and a Dirichlet condition on temperature at $x=b_{1}$ :

$$
\left\{\begin{array}{l}
T_{1,1}^{i_{1}}=T_{b_{1}} \\
\phi_{1, N_{1}}^{i_{1}}=\phi_{b}+h\left(T_{b}-T_{1, N_{1}}^{i_{1}}\right) \\
\text { with } i_{1}=1, n_{1}
\end{array}\right.
$$

In Eq. $19 \phi_{b}, T_{b}$ and $T_{b_{1}}$ are constant heat flux and temperatures. The vector of unknowns $U_{1}$ is introduced as:

$$
U_{1}=\left[\begin{array}{lllll}
T_{1,1} & \ldots & T_{1, N_{1}} & T_{b} & \phi_{b}
\end{array}\right]^{t}
$$

The constants $T_{b}$ and $\phi_{b}$ are introduced in the vector $U_{1}$ at this level of the study in order to prepare the notations used during the analysis of the coupled problem $\mathcal{P}_{\mathcal{S C H} \mathcal{T}}$. The solutions at time $i_{1} \Delta t_{1}$ and $\left(i_{1}+1\right) \Delta t_{1}$ are linked by:

$$
U_{1}^{i_{1}+1}=M_{1}\left(\mathcal{F}_{1}, h\right) U_{1}^{i_{1}}
$$

In Eq. 21, $M_{1}\left(\mathcal{F}_{1}, h\right)$ is the discretisation operator of Eq. 16 augmented by boundary conditions Eq. 19:

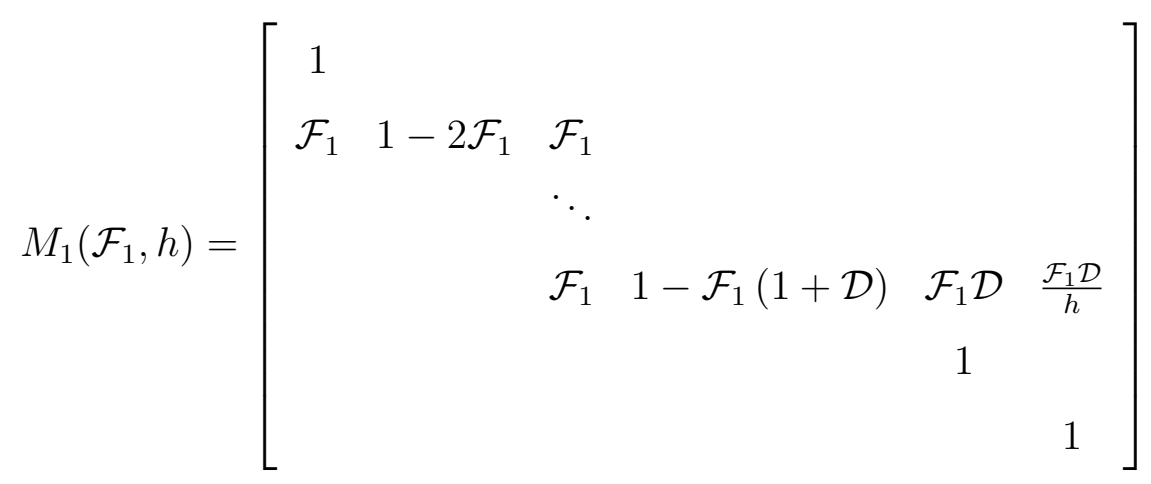

where $\mathcal{D}$ is a Biot like number based on the mesh size in domain $\Delta x_{1}$, the conductivity $\lambda_{1}$ and the coupling relaxation parameter $h$ used in Eq. 8:

$$
\mathcal{D}=\frac{h \Delta x_{1}}{\lambda_{1}}
$$


The value of the spectral radius $\rho\left(M_{1}\left(\mathcal{F}_{1}, h\right)\right)$ of the operator $M_{1}\left(\mathcal{F}_{1}, h\right)$ determines the stability of the discretization (Hirsch, 1988; Allaire and Craig, 2007). Indeed, a requirement for a stable numerical scheme is:

$$
\rho\left(M_{1}\left(\mathcal{F}_{1}, h\right)\right) \leq 1
$$

When the dimension of matrix $M_{1}\left(\mathcal{F}_{1}, h\right)$ is sufficiently small (i.e. when the number of discretization nodes $N_{1}$ is small), the eigen values of $M_{1}$ can be expressed analytically. For $N_{1}=4$, the condition of stability (Eq. 24) gives a bounded region of stability for $\mathcal{D}$ :

$$
\mathcal{D} \leq \frac{\mathcal{F}_{1}^{3}-12 \mathcal{F}_{1}^{2}+20 \mathcal{F}_{1}-8}{-3 \mathcal{F}_{1}^{3}+8 \mathcal{F}_{1}^{2}-4 \mathcal{F}_{1}}
$$

The stability limit of $\mathcal{D}$ is a function of the Fourier number $\mathcal{F}_{1}$ : above $\mathcal{F}_{1}=0.5$, the scheme is always unstable as expected for an explicit formulation. When $\mathcal{F}_{1}$ goes to $0\left(\Delta t_{1}\right.$ tends to 0$)$, the upper limit $\mathcal{D}_{u}$ of $\mathcal{D}$ is $+\infty$ : the scheme becomes unconditionally stable. On the interval $\left.\left.\mathcal{F}_{1} \in\right] 0,0.5\right]$, the derivative $\partial \mathcal{D}_{u} / \partial \mathcal{F}_{1}$ is negative. The use of larger time steps (increasing $\mathcal{F}_{1}$ ) reduce the range of $\mathcal{D}$ leading a stable scheme. Decreasing $\mathcal{D}$ also has a positive effect on convergence (see sub-section 3.5) so that the optimum point for CHT in this case is $\mathcal{F}_{1}=0.5$ and $\mathcal{D}=2.33$. Figure 3 compares the analytical evolution of $\mathcal{D}_{u}$ given by Eq. 25 for $\left.\left.\mathcal{F}_{1} \in\right] 0,0.5\right]$ and numerical solutions obtained with a discrete procedure. The discrete procedure is validated with the case $N_{1}=4$ (Fig. 3 ) and used to measure the sensibility of the stability domain to $N_{1}$. Results show that the curve $\mathcal{D}_{u}\left(\mathcal{F}_{1}\right)$ weakly depends on the number of nodes $N_{1}$ : the case $N_{1}=51$ leads to the same stability limit as $N_{1}=4$. When the number of nodes $N_{1}$ increases, the optimal point for convergence speed goes to $\mathcal{F}_{1}=0.5$ and $\mathcal{D} \approx 2$. 


\subsubsection{Stability analysis of the coupled discretizations}

Now that the stability of domain \#1 with a mixed boundary condition has been studied, the stability of the coupled problem $\mathcal{P}_{\mathcal{S C H} \mathcal{T}}$ is investigated. Between the synchronizations $n_{c p l}$ and $n_{c p l}+1$, solutions $U_{1}$ and $U_{2}$ of domains \#1 and \#2 are advanced in time using discretization operators $M_{1}\left(\mathcal{F}_{1}, h\right)$ and $M_{2}\left(\mathcal{F}_{2}\right)$ similar to the definition given in Eq. 22 :

$$
\left\{\begin{aligned}
U_{1}^{n_{c p l}+1} & =\left(\prod_{n=1}^{n_{1}} M_{1}\right) U_{1}^{n_{c p l}}=M_{1}^{n_{1}} U_{1}^{n_{c p l}} \\
U_{2}^{n_{c p l}+1} & =\left(\prod_{n=1}^{n_{2}} M_{2}\right) U_{2}^{n_{c p l}}=M_{2}^{n_{2}} U_{2}^{n_{c p l}}
\end{aligned}\right.
$$

where the numbers of iterations $n_{1}$ and $n_{2}$ between two synchronization events are linked to time-steps of domains \#1 and \#2:

$$
\begin{aligned}
& n_{1}=\alpha \tau_{1} / \Delta t_{1} \\
& n_{2}=\alpha \tau_{2} / \Delta t_{2}
\end{aligned}
$$

The characteristic time scales $\tau_{1}$ and $\tau_{2}$ in Eq. 27 are chosen in order to mimic

the behavior of CHT of the turbine vane given in Tab. 5. Thus, $\tau_{1}=\frac{\rho_{1} C_{1} b_{1}^{2}}{\lambda_{1}}$ is a diffusion time in domain $\# 1$ while $\tau_{2}$ is a convection time taken in the condition of the blade simulations. For the same reason, the time-step $\Delta t_{1}$ is obtained with a Fourier number $\mathcal{F}_{1}$ equal to 0.5 while $\Delta t_{2}$ is of the order of the time-step in LES computations, viz. $\mathcal{F}_{2}<<0.5$ (the CFL stability limit associated to acoustic waves is usually much more restrictive than diffusion in compressible LES).

Based on Eq. 26, the discretization of the whole system can be expressed as:

$$
U^{n_{c p l}+1}=\mathcal{M}(\alpha, h) U^{n_{c p l}}
$$


where $U$ is a composition of the nodal temperatures in the domains and heat fluxes at the interface. The value of the spectral radius $\rho(\mathcal{M}(\alpha, h))$ of the operator $\mathcal{M}(\alpha, h)$ determines the stability of the discretization (Hirsch, 1988; Allaire and Craig, 2007). Figure 4 gives the stability map of the operator $\mathcal{M}(\alpha, h)$ for $\alpha$ in $[0.001,3]$ and $\mathcal{D}=h \Delta x_{1} / \lambda_{1}$ from 0 (Neumann boundary condition) to 2.3. Above a threshold $\mathcal{D}=\mathcal{D}_{m}$, the mixed condition is stable. As shown on Fig. $4, \mathcal{D}_{m}$ is a function of $\alpha$ : if the CSTP is sufficiently small $(\alpha<0.02)$, the Neumann condition is stable. Then, until $\alpha=0.5$, the value of $\mathcal{D}$ that ensures numerical stability increases with $\alpha$. Beyond this point, $\mathcal{D}_{m}$ remains approximatively constant when the CSTP increases. The stability domain is also limited by an upper bound $\mathcal{D}_{M}$ (Fig. 4). The limit of $\mathcal{D}_{M}$ when $\alpha$ increases is $\mathcal{D}_{u} \approx 2$ obtained during the stability analysis of domain \#1 alone.

As a summary, the stability analyses performed for a simple 1D problem have pointed out a stability domain in terms of $(\alpha, \mathcal{D})$ where $\alpha$ stands for the frequency between two successive updates of the coupled boundary conditions and $\mathcal{D}=h \Delta x_{1} / \lambda_{1}$ where $h$ is a coupling relaxation factor defined in the mixed boundary condition (Eq. 13). For a given value of the coupling synchronization time parameter $\alpha$, a range of $h$ allows numerical stability of the coupled scheme. This range of CRP is bounded by an upper limit determined by the solid discretization whereas the lower bound is linked to the coupling scheme.

An important information not accessible from these analyses still misses: since the aim of the film-cooled turbine vane simulations is to find a stationary thermal state of the blade in a reduced computational time, it is crucial to 
understand the effect of the CSTP $\alpha$ and of the CRP $h$ on convergence. Subsection 3.5 presents sensitivity analyses obtained on the blade configuration when varying $\alpha$ and $h$.

\section{Application of the coupled strategy to the T120D blade}

This section presents the application of the conjugate heat transfer $(\mathrm{CHT})$ strategy to the T120D blade. After describing the configuration, an adiabatic computation is analyzed. It show the potential of the LES to predict accurately complex turbomachinery flows. Then, CHT results are presented. Finally the performance of the CHT methodology is discussed.

\subsection{Description of the T120D blade}

The T120 cascades were designed by Rolls Royce Deutschland for the European project AITEB (Haselbach and Schiffer, 2007) (Aerothermal Investigations on Turbine Endwalls and Blades). The experiments were conducted in the High-speed Cascade Wind Tunnel of the Institute of Jet Propulsion (Sturm and Fottner, 1985; Homeier and Haselbach, 2005; Gomes and Niehuis, 2009).

The highly-loaded high-pressure turbine airfoil of the T120 cascades was designed in order to have a large separation on the pressure side. Therefore the profile presents a strong concave curvature on the pressure side starting shortly after the leading edge. Together with the high pitch to chord ratio relatively strong adverse pressure gradients are present on the pressure side resulting in flow separation. Typically for high-pressure turbine blades, a high change of direction is imposed on the flow. The blade is designed for 
high exit Mach numbers. The aerodynamical and geometrical data at design conditions are listed in Tab. 3.

The film cooling device of the T120D blade is composed of three jet rows located on the pressure side in the region of the main flow separation (Fig. 5). The first row of jets is placed near the stagnation point and has cylindrical holes with compound angle against the main stream. This first row is located to protect the leading edge region which has the highest heat transfer rate over the entire airfoil (Han et al., 2000). The second jet row contains fanshaped holes with zero compound angle located at approximately $20 \%$ of the axial chord length. The second row is supposed to re-energize the boundary layer of the main stream and therefore reduce the flow separation. A third row of cylindrical holes is placed at approximately $35 \%$ of the axial chord. This last row slightly diminishes the separation bubble on the pressure side. The temperature difference between the mainstream $\left(T_{1}^{t}=333.15 \mathrm{~K}\right)$ and cooling $\left(T_{c}^{t}=303.15 \mathrm{~K}\right)$ flows is limited to $30 \mathrm{~K}$ to facilitate measurements. The blade is made of plexiglass with a low conductivity of $0.184 \mathrm{~W} \cdot \mathrm{m}^{-1} \cdot \mathrm{K}^{-1}$ that make the CHT problem difficult to treat. Experimental results include pressure data on the blade suction and pressure sides as well as temperature measurement on the pressure side (Gomes and Niehuis, 2009).

\subsection{Computational domains}

The computational domains for the fluid and the structure contain one spanwise pitch of the film cooling hole pattern corresponding to $8 \mathrm{~mm}$ along the $z$-axis (Fig. 5), with periodicity enforced on each side. This simplification neglects end-wall effects but retains the three-dimensionality of the flow and greatly reduces the number of tetrahedral cells required to model the blade: 
about 6.5 million cells for the fluid and 600,000 for the solid. For the fluid region, periodicity condition is also assumed in the $y$-direction. Specific care is devoted to the tetrahedral cell isotropy in the wall regions; the maximum values of grid spacings on the blade surface expressed in wall units are about $d x^{+} \approx d y^{+} \approx d z^{+} \approx 40$. In the dilution zone, grid spacings are smaller than 5 wall units. Such resolutions are fine enough to capture major aerodynamic events at the wall reasonably well. The size of one spanwise pitch of hole pattern allows these grid spacings with a moderate number of cells. As shown in Fig. 5, the three film-cooling holes and the plenum used to inject the cooling air are also included in the fluid domain. Hence, the distribution of mass flow rate within the holes is a result of the simulations. The skin meshes are the same for the fluid and the solid so that no interpolation error is introduced at this level when CHT is simulated. The computational domains for both fluid and solid parts are considered fine enough to assess the coupling methodology in a reasonable time.

\subsection{Adiabatic computation of the T120D blade}

The description of the LES results with adiabatic wall conditions justifies the use of LES for CHT. The main flow structures are first emphasized before comparing computational results and experimental data.

Figure 6 depicts an instantaneous snapshot of vorticity (left) and a field of

mixture fraction showing the path of cooling air in the main stream (right). The LES predicts an intense turbulence intensity and mixing in the region of the three jets. Downstream from the jets, the strong acceleration on the pressure side relaminarizes the flow and forces the cooling air against the blade surface. At the beginning of the suction side, the boundary layer is 
rather laminar. Then, the flow accelerates up to supersonic velocities. A weak shock appears at a reduced abscissa of about 0.75 and destabilizes the boundary layer. Vortex shedding develops behind the blade.

Figure 7 shows that jet 1, directly exposed to the main stream, mixes rapidly with hot gases. Protected by the concave shape of the blade and by the first jet, the cooling air of the second hole penetrates more into the flow until it mixes with the third jet. Jet 3 is aligned with the main stream and remains coherent until it impacts the blade in a region between reduced abscissa of 0.5 to 0.6 .

The adiabatic LES results are compared to the experiment in terms of pressure profiles on the blade (Fig. 8) and of temperature profiles on the pressure side (Fig. 9). Pressure fields are displayed in terms of isentropic Mach numbers $M_{i s}(\mathbf{x})$ computed by:

$$
M a_{i s}(\mathbf{x})=\sqrt{\frac{2}{\gamma-1}\left[\left(\frac{P_{1}^{t}}{P_{w}^{t}(\mathbf{x})}\right)^{\frac{\gamma-1}{\gamma}}-1\right]}
$$

where $\gamma$ is the isentropic coefficient, $P_{1}^{t}$ and $P_{w}^{t}(\mathbf{x})$ are the total pressure of the mainstream and at the wall at location $\mathbf{x}$. Figure 8 displays a time-averaged distribution of isentropic Mach number obtained by LES and experimentally at static pressure tap locations. The flow strongly accelerates on the suction side up to supersonic conditions $\left(M a_{i s}(\mathbf{x})>1\right)$ and features a weak shock at about $75 \%$ of the axial chord. On the pressure side, the flow is exposed to strong adverse pressure gradients shortly downstream of the stagnation point. The flow separates at a reduced abscissa of about 0.2 (position of the second jet) reattaching at about 0.45 , after the third jet. After reattachment, the flow is strongly accelerated leading to values for the acceleration parameter 
that are beyond common relaminarization limits (Mayle, 1991). Although the shock position on the suction side is not perfectly captured, the overall agreement between LES and experimental results is fair.

Table 4 compares numerical and experimental values of the total mass flow rate of air $Q=\sum_{j} Q_{j}$ passes through the three holes as well as of the blowing ratios $M_{i}$ of the jets. Proper descriptions of the mass flow rate and blowing ratio of each jet are crucial to predict heat transfer with a good accuracy. LES gives a good approximation of the total mass flow rate across the holes. The numerical simulation also gives the distribution of air among the three jets, not available experimentally. Since the total coolant pressure is the same for all three coolant rows, the mass flow rate is almost equally distributed between the holes. Blowing ratios are expressed in terms of velocity $U$ and density $\rho$ as:

$$
M_{j}=\frac{\rho_{j} U_{j}}{\rho_{\infty} U_{\infty}}, j=1,2,3
$$

where $j$ refers to jet quantities and $\infty$ to local main stream values. Equal coolant mass flow $Q_{j}=\rho_{j} U_{j}$ per hole is assumed for the determination of experimental blowing ratios of Tab. 4 and the LES validates this hypothesis. The agreement between experimental and numerical blowing ratios is rather good (Tab. 4).

Wall temperatures $T_{w}^{t}(\mathbf{x})$ are presented in Fig. 9 in terms of cooling efficiency defined by:

$$
\Theta(\mathbf{x})=\frac{T_{1}^{t}-T_{w}^{t}(\mathbf{x})}{T_{1}^{t}-T_{c}^{t}}
$$

where $T_{1}^{t}$ and $T_{c}^{t}$ are the total temperatures at the inlets of the mainstream and plenum respectively. Time and spanwise averaged cooling efficiencies for 
adiabatic LES and RANS computations as well as experimental results are plotted as function of relative axial chord on Fig. 9. The RANS computation is done with Fluent using a $k-\omega / S S T$ turbulence model (Menter, 1993). As expected, in the region of the jets (reduced abscissa up to 0.45 ) the cooling efficiencies obtained with the adiabatic simulations are lower than the experimental values: adiabatic temperature fields over-predict the real one. Downstream of the impact of the jets on the blade, the adiabatic LES fits the experimental level of $\Theta$ whereas the RANS computation over-estimates it. In the experience, the film of colder air that forms after the interaction between the jets and the surface of the blade maintains the wall temperature close to adiabatic one. Hence, the LES captures fairly well the air mass flow through the jets as well as the mixing of the cooling air with the main stream. That is not the case for the RANS simulation. Indeed, even if the RANS computation reproduces the real air mass flow rates ejected by the holes, the simulation does not describe mixing correctly. As a result, the jets remain coherent on a too long distance without mixing with the hot stream and too much cold air impacts the blade surface.

Both LES and RANS simulations exhibit a non-physical peak of $\Theta$ near the trailing edge. This peak is due to an over-expansion near the trailing edge which does not appear in the experiment. The round trailing edge of the T120D blade profile and a lack of resolution in this region where there is a very strong acceleration of the flow cause this difficulty in the computation (Denton and Dawes, 1999; Mei and Guha, 2005).

From these results, LES appears to be a good candidate to treat $\mathrm{CHT}$ problems in complex turbomachinery flows. 


\subsection{Coupled computation of the T120D blade}

This sub-section presents a fully coupled simulation of the T120D blade obtained with a two-step methodology:

1. Initialization of the coupled calculation that includes:

- a converged adiabatic fluid simulation (presented in sub-section 3.3),

- a converged solid computation with imposed boundary temperatures given by the adiabatic fluid solution,

2. Coupled simulation with a parallel coupling strategy (PCS on Fig. 2).

The maximum value of the Biot mesh number $\mathcal{D}=h \Delta x_{s} / \lambda_{s}$ that gives a stable mixed condition for the solid discretization of the blade (Eq. 13) with a Fourier number of 0.5 is $\mathcal{D}_{u} \approx 1.8$. The solid mesh size $\Delta x_{s}$ used to compute $\mathcal{D}_{u}$ corresponds to the discretization size at the fluid / solid interface (i.e min $\Delta x$ for the solid in Tab. 5). The conductivity of the solid media is given in Tab. 1 . It is interesting to note that the value $\mathcal{D}_{u} \approx 2$ highlighted in sub-section 2.2 for $1 D$ simple problem is a good approximation for the complex $3 D$ problem. As a result, the values of the coupling synchronization time parameter $\alpha$ and of the coupling relaxation parameter $h$ used for the CHT simulation are chosen so that the point $(\alpha, h)$ belongs to the stability domain of the simple $1 D$ problem $\mathcal{P}_{\mathcal{S C H}}$ (Fig. 4) presented in section 2. These coupling parameters correspond to $\left(\alpha_{1}, h_{2}\right)$ in Tab. 6 .

The coupled simulation was performed on 32 processors of an IBM JS21. The synchronization in CPU time (Eq. 12) was reached with 30 processors allocated to the fluid solver and 2 for the solid one. The converged 
state is obtained in 10 characteristic solid time scales $\tau_{s}$ (Fig.11) and requires about $4800 \mathrm{CPU}$ hours. As a previous study on this configuration has shown that the pressure distribution over the blade is not affected by the coupling (Duchaine et al., 2008), the analysis focuses on thermal aspects.

At the converged state, the net heat flux through the blade reaches zero (i.e. the mean temperature of the blade is stabilized, Fig. 11). Figure 9 shows measurements, adiabatic and coupled results of the cooling efficiency $\Theta(x)$ spanwise and time averaged along pressure side. As mentioned previously, the adiabatic temperature field (solid line) over-predicts the real one. The main contribution of conduction throughout the blade is to reduce the wall temperature on the pressure side and thus to increase $\Theta(x)$ (dot dashed line). The global form of the reduced temperature from the coupled simulation matches the experimental data correctly. Differences in the levels are explained by the insufficient wall resolution used for the LES computation as well as to the experimental difficulties and uncertainties for temperature measurements and processing (i.e. spanwise average). The strong acceleration caused by the blade induces large thermal gradients not-well resolved by the simulation that leads to an underestimation of the thermal fluxes as well as to non-physical values of cooling efficiency at the trailing edge.

Figure 10 compares experimental and numerical cooling efficiency fields on a $2 \mathrm{D}$ plot of the pressure side. The computation matches the experimental visualization. Figure 10 evidences the thermal effects of the cooling jets on the vane. Jet 1 is folded back against the surface by the main stream but detaches rapidly and mixes with the hot gases due to the curvature of the blade. Downstream of the second hole, a streak with higher efficiency and 
a spot with enhanced cooling close to the ejection location indicate a partially attached jet. A streak with a lower surface temperature is also visible downstream of the third jet. Jet 3 seems to be the most active in the cooling process: it protects the blade from the hot stream until a reduced abscissa of 0.5 and it impacts the vane at reduced abscissa between 0.5 and 0.6 . The curvature of the pressure side induces a slack film coverage: the concave shape of the surface spreads the cooling air laterally along the spanwise direction, as explained by Schwarz and Goldstein (Schwarz and Goldstein, 1989).

\subsection{Efficiency analyses of the coupled strategy}

This last sub-section analyzes the efficiency of the coupling methodology with regard to the adjustable parameters used in the boundary conditions, the coupling synchronization time parameter $\alpha$ and the coupling relaxation parameter $h$ :

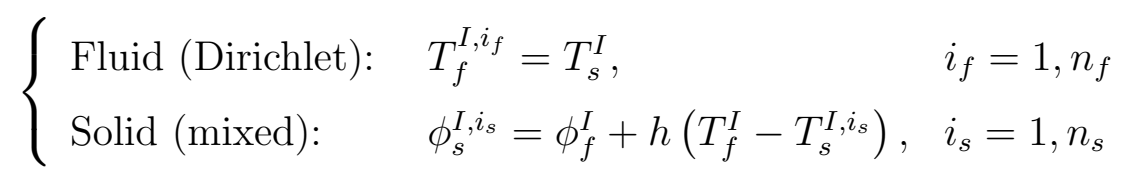

The number of iterations of fluid $\left(n_{f}\right)$ and solid $\left(n_{s}\right)$ solvers between two consecutive updates of boundary conditions are:

$$
\begin{aligned}
& n_{f}=\alpha \tau_{f} / \Delta t_{f} \\
& n_{s}=\alpha \tau_{s} / \Delta t_{s}
\end{aligned}
$$

In Eq. 33, $\Delta t_{f}$ and $\Delta t_{s}$ are the time-steps of the fluid and solid solvers respectively. $\tau_{f}$ and $\tau_{s}$ are characteristic times of the flow and solid phenomena. The different values used for the CSTP $\alpha_{k}$ and CRP $h_{k}$ in this section are summarized in Tab. 6. As the implementation of SCS is simpler than PCS, SCS is preferred for efficiency analyses. As the formulations for SCS 
and PCS are different, it is useful to check whether the implementations of these strategies give the same results: Fig. 11 compares $\operatorname{SCS}\left(\alpha_{1}, h_{2}\right)$ and $P C S\left(\alpha_{1}, h_{2}\right)$ and shows that both strategies converge to the same thermal state at the same rate. As a reference for computational costs and restitution times, one solid characteristic time $\tau_{s}$ requires almost a total of $295 \mathrm{CPU}$ hours for the LES and the heat conduction solvers on a SGI Altix ICE8200.

To evaluate the impact of $h$ in Eq. 32 on restitution times for the T120D CHT problem, three computations with SCS are compared: $S C S\left(\alpha_{2}, h_{1}\right)$, $S C S\left(\alpha_{2}, h_{2}\right)$ and $S C S\left(\alpha_{2}, h_{3}\right)$ (Tab. 6). Figure 12a depicts the evolutions of the mean temperature in the blade as a function of reduced solid time $t_{s} / \tau_{s}$ for these simulations. The retained values $h_{1}, h_{2}$ and $h_{3}$ with $\alpha_{2}$ give stable coupled schemes that converge to the same thermal steady state. Figure 4 shows that the predictions from the $1 D$ problem $\mathcal{P}_{\mathcal{S C H}}$ are not directly applicable to the complex $3 D$ CHT case: following the $1 D$ analysis, the points $\left(\alpha_{2}, h_{2}\right)$ and $\left(\alpha_{2}, h_{3}\right)$ are stable while the point $\left(\alpha_{2}, h_{1}\right)$ is not stable. The convergence rate is influenced by the choice of the CRP : simulations converge faster for slower values of $h$ (Fig. 12a). Indeed from $h_{3}$ to $h_{1}$ the CPU time needed to reach a steady state is divided by approximatively 2.5 .

As done for the CRP $h$, three computations with SCS are then compared to evaluate the effect of the CSTP $\alpha$ in Eq. 33 on convergence: $S C S\left(\alpha_{1}, h_{2}\right)$, $S C S\left(\alpha_{2}, h_{2}\right)$ and $S C S\left(\alpha_{3}, h_{2}\right)$ (Tab. 6). Figure 12b shows the evolutions of the mean temperature in the blade as a function of reduced solid time $t_{s} / \tau_{s}$ for these simulations. The numerical schemes obtained with $\alpha_{1}, \alpha_{2}$ and $\alpha_{3}$ combined with $h_{2}$ are stable and converge to the same thermal steady state. Fig. 12b shows that the choice of the CSTP directly affects the convergence 
rate of the coupled simulations. Small values of $\alpha$ enhances the restitution times of the computations: high update frequencies of interface variables allow the computations to follow the steepest descent to converged state.

To conclude, good convergence rates are obtained for small values of both the CSTP $\alpha$ and CRP $h$ within the stability domain. When $\alpha$ is sufficiently small, SCS becomes inefficient compared to PCS. Indeed, below a certain value of the CSTP, the computational time due to the resolution of fluid and solid equations is smaller than the management times due to launching of the codes as well as initialization and finalization of the computations (reading of meshes and initial solutions, writing of final solutions ...). PCS allows to avoid these restart steps and is therefore recommended. It is also important to note that when the CSTP $\alpha$ becomes too small (i.e. decreasing the number of iterations of the solvers), the execution time of the fluid and solid solvers between two successive updates of boundary conditions can become lower than the time requested to data exchanges. Such a configuration is not acceptable for the efficiency of the method and a balance is needed to fix the CSTP.

\section{Conclusion}

The thermal loads on gas turbine components controls life-time of the system. Numerical predictions of conjugate heat transfer raise problems which make the simulations difficult to compute:

- The flows are very complex: three-dimensional unsteady turbulent effects, mixing of flows with different properties, high speeds with com- 
pressibility effects (with large variations of Mach number up to supersonic conditions),

- Distinct physics must be coupled with very different length and time scales,

- The size of the computations requires parallel computations to reduce restitution times.

In the present study, a coupling strategy for conjugate heat transfer with Large Eddy Simulation (LES) is proposed and applied to a film-cooled turbine vane experimentally tested at the Institute of Jet Propulsion. The methodology is based on a partitioned approach: a LES solver exchanges information with a heat transfer code within solids via a supervisor. It is shown that the LES solver is well adapted to capture the flow that develops around the film-cooled turbine blade.

The coupling methodology is first assessed in term of stability and efficiency. At the fluid/solid interface, a mixed condition on wall temperature and heat fluxes is written based on a coupling relaxation parameter $h$. A stability analysis on a simplified one-dimensional problem allows the determination and optimization of coupling parameters. Two parameters control both the stability and cost of the coupled simulations: the mesh Biot number of the solid domain $\mathcal{D}=h \Delta x_{s} / \lambda_{s}$ (where $\Delta x_{s}$ and $\lambda_{s}$ are respectively the solid discretization and the solid conductivity) and the coupling synchronization time parameter $\alpha$ which defined the time between two coupling events. The coupled tool is ported on massively parallel architectures and applied to an experimental film-cooled turbine vane. Results demonstrate both the 
efficiency of the parallel implementation and the quality of the results. Thermal convergence of the blade is accelerated by high frequencies of exchanges between the codes (small values of the coupling synchronization time parameter $\alpha$ ). Coupled and non-coupled simulations are compared to experimental results and discussed in terms of cooling efficiency and flow structures.

The comparison between adiabatic LES and measurements is good for the isentropic Mach number along the blade. Reasonable aerodynamic predictions are a necessary condition to reproduce the blade skin temperature field. As the LES computation gives a correct air flow distribution within the cooling system and reproduces the mixing between the hot stream and the cooling flow, the trends of computed cooling efficiency agree reasonably well with experimental data. Discrepancies in the levels are mainly due to a lack of resolution in the fluid flow near the wall. Finally, the coupled simulation is used to describe the role of the three rows of cooling jets in heat transfer.

Further studies will concern the analyze of mesh resolution as well as the use of thermal wall model to enhance thermal predictions. The effect of the number of spanwise pitch of the film cooling hole pattern including jet to jet interactions will also be investigated.

\section{Acknowledgements}

A large part of numerical simulations have been conducted on the computers of the French National Computing Center (CINES) in Montpellier. Part of this work was performed at the 2008 Stanford CTR Summer Pro-

gram. The help of R. Gomes from the University of the Federal Armed Forces Munich and of the AITEB-1 and AITEB-2 consortium who provided exper- 
imental results is gratefully acknowledged. Dr. N. Lamarque is gratefully acknowledged for the various discussions on numerics. 


\section{References}

B. Lakshminarayana, Fluid Dynamics and Heat Transfer of Turbomachinery, John Wiley \& Sons, Inc., New York, NY, USA, 1996.

A. H. Lefebvre, Gas Turbines Combustion, Taylor \& Francis, 1999.

R. Schiele, S. Wittig, Gas Turbine Heat Transfer: Past and Future Challenges, J. Prop. Power 16 (4) (2000) 583-589.

M. G. Dunn, Convective heat transfer and aerodynamics in axial flow turbines, J. Turbomach. 123 (2001) 637-686.

R. S. Bunker, Gas turbine heat transfer: Ten remaining hot gas path challenges, J. Turbomach. 129 (2007) 193-201.

P. Sagaut, Large Eddy Simulation for incompressible flows, Scientific computation series, Springer-Verlag, 2000.

K. Mahesh, G. Constantinescu, P. Moin, A numerical method for large-eddy simulation in complex geometries, J. Comput. Phys. 197 (1) (2004) 215240, ISSN 0021-9991.

T. Poinsot, D. Veynante, Theoretical and Numerical Combustion, R.T. Edwards, 2nd edition., 2005.

S. Acharya, M. Tyagi, A. Hoda, Flow and Heat Transfer Predictions for Film Cooling, Heat transfer in gas turbine systems. Annals of the New York Academy of Sciences 934 (2001) 110-125. 
A. Azzi, D. Lakehal, Perspectives in Modeling Film Cooling of Turbines Blades by Transcending Conventional Two-Equation Turbulence Models, J. Turbomach. 124 (2002) 472-484.

A. Rozati, Large Eddy Simulation of Leading Edge Film Cooling: Flow Physics, Heat Transfer, and Syngas Ash Deposition, Ph.D. thesis, Virginia Polytechnic Institute and State University, 2007.

G. Boudier, L. Y. M. Gicquel, T. Poinsot, D. Bissières, C. Bérat, Comparison of LES, RANS and Experiments in an Aeronautical Gas Turbine Combustion Chamber, Proc. Combust. Inst. 31 (2007) 3075-3082.

K. H. Kao, M. S. Liou, Application of Chimera/Unstructured Hybrid Grids for Conjugate Heat Transfer, AIAA Journal 35 (9) (1997) 1472-1478.

Z. X. Han, B. Dennis, G. Dulikravich, Simultaneous prediction of external flow-field and temperature in internally cooled 3-D turbine blode material, Int. J. Turbo Jet-Eng. 18 (2001) 47-58.

F. Rahman, J. A. Visser, R. M. Morris, Capturing Sudden Increase in Heat Transfer on the Suction Side of a Turbine Blade Using a Navier-Stokes Solver, J. Turbomach. 127 (3) (2005) 552-556.

J. Luo, E. H. Razinsky, Conjugate heat transfer analysis of a cooled turbine vane using the V2F turbulence model, J. Turbomach. 129 (4) (2007) 773781.

V. Ganesan, Non-Reacting and Reacting Flow Analysis in an Aero-Engine Gas Turbine Combustor Using CFD, in: SAE 2007 World Congress, 200701-0916, Detroit, Michigan, USA, 2007. 
A. Heselhaus, D. T. Vogel, Numerical simulation of turbine blade cooling with respect to blade heat conduction and inlet temperature profiles, in: ASME, SAE, and ASEE, Joint Propulsion Conference and Exhibit, 31st, AIAA-1995-3041, San Diego, CA, USA, 1995.

D. L. Sondak, D. J. Dorney, Simulation of Coupled Unsteady Flow and Heat Conduction in Turbine Stage, J. Prop. Power 16 (6) (2000) 1141-1148.

E. Papanicolaou, D. Giebert, R. Koch, A. Schulz, A conservation-based discretization approach for conjugate heat transfer calculations in hot-gas ducting turbomachinery components, Int. J. Heat and Mass Transfer 44 (18) (2001) 3413-3429.

V. K. Garg, Heat transfer research on gas turbine airfoils at NASA GRC, Int. J. Heat Fluid Flow 23 (2) (2002) 109-136.

D. Bohn, J. Ren, K. Kusterer, Systematic Investigation on Conjugate Heat Transfer Rates of Film Cooling Configurations, Int. J. Rotating Machinery 2005 (3) (2005) 211-220.

J. J. Alonso, S. Hahn, F. Ham, M. Herrmann, G. Iaccarino, G. Kalitzin, P. LeGresley, K. Mattsson, G. Medic, P. Moin, H. Pitsch, J. Schlüter, M. Svard, E. der Weide, D. You, X. Wu, CHIMPS: a highperformance scalable module for multi-physics simulation, in: 42nd AIAA/ASME/SAE/ASEE Joint Propulsion Conference \& Exhibit, AIAAPaper 2006-5274, 2006.

B. Vreman, B. Geurts, H. H. Kuerten, On the formulation of the dynamic mixed subgrid-scale model, Phys. Fluids 6 (12) (1994) 4057-4059. 
J. H. Ferziger, Large eddy simulations of turbulent flows, AIAA Journal 15 (9) (1977) 1261-1267.

J. Smagorinsky, General circulation experiments with the primitive equations: 1. The basic experiment., Mon. Weather Rev. 91 (1963) 99-164.

S. B. Pope, Turbulent flows, Cambridge University Press, 2000.

P. Chassaing, Turbulence en mécanique des fluides, Analyse du phénomène en vue de sa modélisation à l'usage de l'ingénieur, Cépaduès-éditions, Toulouse, France, 2000.

F. Nicoud, F. Ducros, Subgrid-scale stress modelling based on the square of the velocity gradient, Flow, Turb. and Combustion 62 (3) (1999) 183-200, doi:10.1023/A:1009995426001.

P. Moin, K. D. Squires, W. Cabot, S. Lee, A dynamic subgrid-scale model for compressible turbulence and scalar transport, Phys. Fluids A 3 (11) (1991) 2746-2757, doi:10.1063/1.858164.

G. Erlebacher, M. Y. Hussaini, C. G. Speziale, T. A. Zang, Toward the large-eddy simulation of compressible turbulent flows, J. Fluid Mech. 238 (1992) 155-185.

F. Ducros, P. Comte, M. Lesieur, Large-eddy simulation of transition to turbulence in a boundary layer developing spatially over a flat plate, J. Fluid Mech. 326 (1996) 1-36.

P. Comte, New tools in Turbulence Modelling. Vortices in incompressible 
LES and non-trivial geometries, Springer-Verlag, France, course of Ecole de Physique des Houches, 1996.

D. K. Lilly, A proposed modification of the germano sub-grid closure method, Phys. Fluids 4 (3) (1992) 633-635.

M. Germano, Turbulence: the filtering approach, J. Fluid Mech. 238 (1992) 325-336.

S. Ghosal, P. Moin, The basic equations for the large eddy simulation of turbulent flows in complex geometry, J. Comput. Phys. 118 (1995) 24 37.

C. Meneveau, T. Lund, W. Cabot, A lagrangian dynamic subgrid-scale model of turbulence, J. Fluid Mech. 319 (1996) 353.

T. Poinsot, S. Lele, Boundary conditions for direct simulations of compressible viscous flows, J. Comput. Phys. 101 (1) (1992) 104-129, doi: 10.1016/0021-9991(92)90046-2.

T. Schönfeld, T. Poinsot, Influence of boundary conditions in LES of premixed combustion instabilities, in: Annual Research Briefs, Center for Turbulence Research, NASA Ames/Stanford Univ., 73-84, 1999.

V. Moureau, G. Lartigue, Y. Sommerer, C. Angelberger, O. Colin, T. Poinsot, Numerical methods for unsteady compressible multicomponent reacting flows on fixed and moving grids, J. Comput. Phys. 202 (2) (2005) 710-736, doi:10.1016/j.jcp.2004.08.003. 
S. Mendez, F. Nicoud, Large-eddy simulation of a bi-periodic turbulent flow with effusion, J. Fluid Mech. 598 (2008) 27-65.

A. Roux, L. Y. M. Gicquel, Y. Sommerer, T. J. Poinsot, Large eddy simulation of mean and oscillating flow in a side-dump ramjet combustor, Combust. Flame 152 (1-2) (2008) 154-176.

J. Donea, A. Huerta, Finite Element Methods for Flow Problems, John Wiley \& Sons Inc, New York, 2003.

O. Colin, M. Rudgyard, Development of high-order Taylor-Galerkin schemes for unsteady calculations, J. Comput. Phys. 162 (2) (2000) 338-371.

S. Chemin, Étude des Interactions Thermiques Fluides-Structure par un Couplage de Codes de Calcul, Ph.D. thesis, Université de Reims ChampagneArdenne, 2006.

M. B. Giles, Stability analysis of numerical interface conditions in fluidstructure thermal analysis, Int. J. Numer. Meth. Fluids 25 (4) (1997) 421-436.

E. Radenac, Développement et validation d'une méthode numérique pour le couplage fluide structure en aérothermique instationnaire, Ph.D. thesis, Université Paul Sabatier - Toulouse, 2006.

B. Roe, R. Jaiman, A. Haselbacher, P. H. Geubelle, Combined interface boundary condition method for coupled thermal simulations, Int. J. Numer. Meth. Fluids 57 (3) (2008) 329-354. 
F. Duchaine, S. Mendez, F. Nicoud, A. Corpron, V. Moureau, T. Poinsot, Coupling heat transfer solvers and large eddy simulations for combustion applications, in: Proc. of the Summer Program, Center for Turbulence Research, NASA Ames/Stanford Univ., 57-72, 2008.

C. A. Felippa, K. C. Park, C. Farhat, Partitioned analysis of coupled mechanical systems, Comput. Methods Appl. Mech. Eng. 190 (24-25) (2001) $3247-3270$.

E. Divo, E. Steinthorsson, A. Kassab, Bialecki, An iterative BEM/FVM protocol for steady-state multi-dimensional conjugate heat transfer in compressible flows, Eng. An. Bound. Elements 26 (5) (2002) 447-454.

J. D. Heidmann, A. J. Kassab, E. A. Divo, F. Rodriguez, E. Steinthorsson, Conjugate Heat Transfer Effects on a Realistic Film-Cooled Turbine Blade, in: Proceedings of ASME Turbo Expo 2003, ASME Paper GT2003-38553, Atlanta, Georgia, USA, 2003.

E. Mercier, L. Tesse, N. Savary, 3D Full Predictive Thermal Chain for Gas Turbine, in: 25th International Congress of the Aeronautical Sciences, Hamburg, Germany, 2006.

T. Verstraete, Z. Alsalihi, R. A. V. den Braembussche, Numerical study of the heat transfer in micro gas turbines, J. Turbomach. 129 (4) (2007) $835-841$.

A. Montenay, L. Paté, J. M. Duboué, Conjugate heat transfer analysis of an engine internal cavity, in: Proceedings of ASME Turbo Expo 2000, ASME Paper 2000-GT-282, Munich, Germany, 2000. 
A. Hegab, T. L. Jackson, J. Buckmaster, D. S. Stewart, Nonsteady burning of periodic sandwich propellants with complete coupling between the solid and gas phases, Combust. Flame 125 (1-2) (2001) 1055-1070.

R. G. dos Santos, M. Lecanu, S. Ducruix, O. Gicquel, E. Iacona, D. Veynante, Coupled large eddy simulations of turbulent combustion and radiative heat transfer, Combust. Flame 152 (3) (2008) 387-400.

T. Lagarde, A. Piacentini, O. Thual, A new representation of dataassimilation methods: The PALM flow-charting approach, Q. J. R. Meteorol. Soc. 127 (571) (2001) 189-207.

S. Buis, A. Piacentini, D. Déclat, PALM: A Computational Framework for assembling High Performance Computing Applications, Concurrency and Computation 18 (2) (2005) 231-245.

D. S. Burnett, Finite Element Analysis, Addison-Wesley, 1987.

C. Hirsch, Numerical Computation of Internal and External Flows, vol. 1, John Wiley, New York, 1988.

G. Allaire, A. Craig, Numerical Analysis and Optimization: An Introduction to Mathematical Modelling and Numerical Simulation, Oxford University Press, 2007.

F. Haselbach, P. Schiffer, AITEB - An european research project on aerothermodynamics of turbine endwalls and blades, Int. J. of Therm. Sci. 13 (2) (2007) 97-108. 
W. Sturm, L. Fottner, The High-Speed Cascade Wind-Tunnel of the German Armed Forces University Munich, in: 8th Symposium on Measuring Techniques for Transonic and Supersonic Flows in Cascades and Turbomachines, Genova, Italy, 1985.

L. Homeier, F. Haselbach, Film Cooling of Highly Loaded Blades, in: XVIII International Symposium on Air Breathing Engines (ISABE), AIAA-20051114, Munich, Germany, 2005.

R. A. Gomes, R. Niehuis, Film cooling Effectiveness measurements with periodic unsteady inflow on highly loaded blades with main flow separation, in: Proceedings of ASME Turbo Expo 2009: Power for Sea, Land and Air, Orlando, Florida, USA, 2009.

J. C. Han, S. Dutta, S. V. Ekkad, Gas Turbine Heat Transfer and Cooling Technology, Taylor \& Francis, New York, NY, USA, 2000.

R. E. Mayle, The Role of Laminar-Turbulent Transition in Gas Turbine Engines, J. Turbomach. 113 (1991) 509-537.

F. R. Menter, Zonal Two Equation k-w Turbulence Models For Aerodynamic Flows, in: Fluid Dynamics, Plasmadynamics, and Lasers Conference, 23rd, AIAA-1993-2906, Orlando, FL, USA, 1993.

J. Denton, W. N. Dawes, Computational fluid dynamics for turbomachinery design, in: M. E. Publications (Ed.), Proceedings of the Institution of Mechanical Engineers. Part C, Journal of mechanical engineering science, vol. 213, London, United Kingdom, 107-124, 1999. 
Y. Mei, A. Guha, Implicit numerical simulation of transonic flow through turbine cascades on unstructured grids, in: P. E. Publishing (Ed.), Proceedings of the Institution of Mechanical Engineers. Part A. Journal of power and energy, vol. 219, Bury St Edmunds, United Kingdom, 95-47, 2005.

S. G. Schwarz, R. J. Goldstein, The Two-Dimensional Behavior of Film Cooling Jets on Concave Surfaces, J. Turbomach. 111 (1989) 124-130. 


\section{Tables}

\begin{tabular}{lcc}
\hline Thermal conductivity & $\lambda_{s}$ & $0.184 \mathrm{~W} \cdot \mathrm{m}^{-1} \cdot \mathrm{K}^{-1}$ \\
Heat capacity & $C_{s}$ & $1450 \mathrm{~J} \cdot \mathrm{kg}^{-1} \cdot \mathrm{K}^{-1}$ \\
Density & $\rho_{s}$ & $1190 \mathrm{~kg} \cdot \mathrm{m}^{-3}$ \\
Thermal diffusivity & $D_{s}$ & $1.0710^{-7} \mathrm{~m}^{2} \cdot \mathrm{s}^{-1}$ \\
\hline
\end{tabular}

Table 1: Thermal characteristics of plexiglass.

\begin{tabular}{lcc}
\hline Thermal conductivity & $\lambda$ & $2.610^{-2} \mathrm{~W} \cdot \mathrm{m}^{-1} \cdot \mathrm{K}^{-1}$ \\
Heat capacity & $C p$ & $1015 \mathrm{~J} \cdot \mathrm{kg}-1 \cdot \mathrm{K}^{-1}$ \\
Density & $\rho$ & $0.266 \mathrm{~kg} \cdot \mathrm{m}^{-3}$ \\
Thermal diffusivity & $D_{f}$ & $9.6310^{-5} \mathrm{~m}^{2} \cdot \mathrm{s}^{-1}$ \\
\hline
\end{tabular}

Table 2: Thermal characteristics of Air at $328 \mathrm{~K}$. 


\begin{tabular}{lcc}
\hline Blade height to chord ratio & & 1.5 \\
Pitch to chord ratio & & 1.007 \\
\hline Turning & $\Delta \beta$ & $120^{\circ}$ \\
Inlet angle & $\beta_{1}$ & $138^{\circ}$ \\
\hline Inlet Mach number & $M a_{1}$ & 0.295 \\
Oulet Mach number & $M a_{2}$ & 0.87 \\
Oulet Reynolds number & $R e_{2}$ & 390000 \\
Inlet total pressure & $P_{1}^{t}$ & $26737.0 \mathrm{~Pa}$ \\
Inlet total temperature & $T_{1}^{t}$ & $333.15 \mathrm{~K}$ \\
Cooling total pressure & $P_{c}^{t}$ & $29143.3 \mathrm{~Pa}$ \\
Cooling total temperature & $T_{c}^{t}$ & $303.15 \mathrm{~K}$ \\
Outlet static pressure & $P_{2}$ & $16325 \mathrm{~Pa}$ \\
\hline
\end{tabular}

Table 3: Experimental settings for T120D blade.

\begin{tabular}{cccccccc}
\hline & $\begin{array}{c}Q_{1} \\
{\left[g \cdot s^{-1}\right]}\end{array}$ & $\begin{array}{c}Q_{2} \\
{\left[g \cdot s^{-1}\right]}\end{array}$ & $\begin{array}{c}Q_{3} \\
{\left[g \cdot s^{-1}\right]}\end{array}$ & $\begin{array}{c}Q=\sum_{j} Q_{j} \\
{\left[g \cdot s^{-1}\right]}\end{array}$ & $M_{1}$ & $M_{2}$ & $M_{3}$ \\
\hline \hline Experiment & - & - & - & 0.250 & 2.03 & 2.33 & 3.78 \\
LES & 0.083 & 0.090 & 0.075 & 0.248 & 2.1 & 2.3 & 3.0 \\
\hline
\end{tabular}

Table 4: Mass flow rate of air through the three jets $Q_{j}$ and blowing ratios (Eq. 30) of each jet $M_{j}$ of the T120D blade.

\begin{tabular}{ccc}
\hline & Fluid & Solid \\
\hline \hline $\min \Delta x$ & $1.2310^{-4} \mathrm{~m}$ & $1.4110^{-4} \mathrm{~m}$ \\
$\Delta t$ & $9.6510^{-8} \mathrm{~s}$ & $1.7410^{-2} \mathrm{~s}$ \\
$\tau$ & $1.210^{-1} \mathrm{~s}$ & $233.6 \mathrm{~s}$ \\
\hline
\end{tabular}

Table 5: Minimum length, time-steps and characteristic times of fluid and solid discretisation for the T120D blade.

$$
\begin{array}{ccc}
\hline \alpha_{1}=0.1 & \alpha_{2}=0.85 & \alpha_{3}=5.00 \\
h_{1}=50 & h_{2}=100 & h_{3}=200 \\
\hline
\end{array}
$$

Table 6: Values of the coupling synchronization time parameter and coupling relaxation parameter used in section 3 . 


\section{FIGURES}

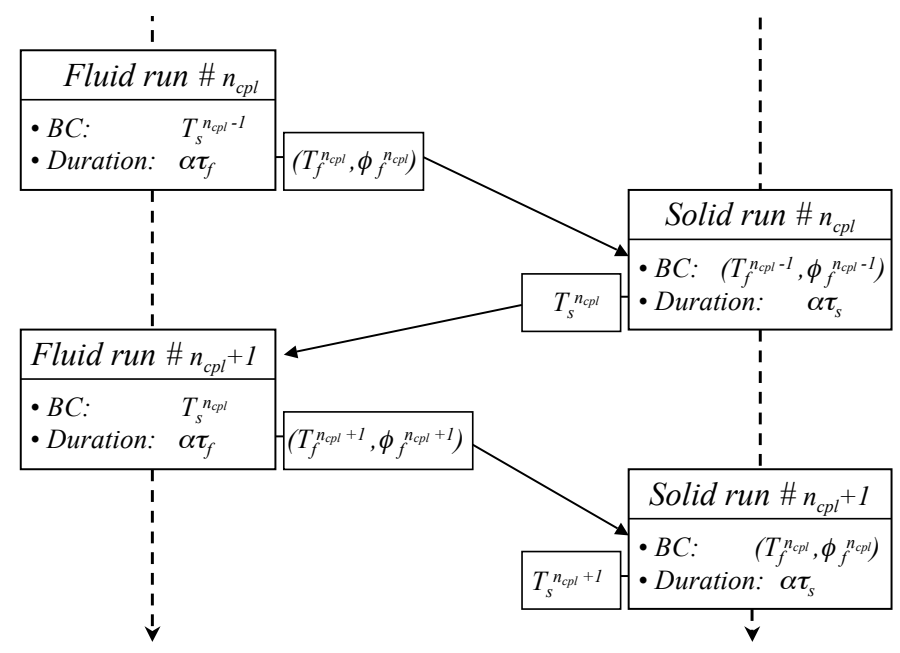

Figure 1: Sequential coupling strategy SCS .

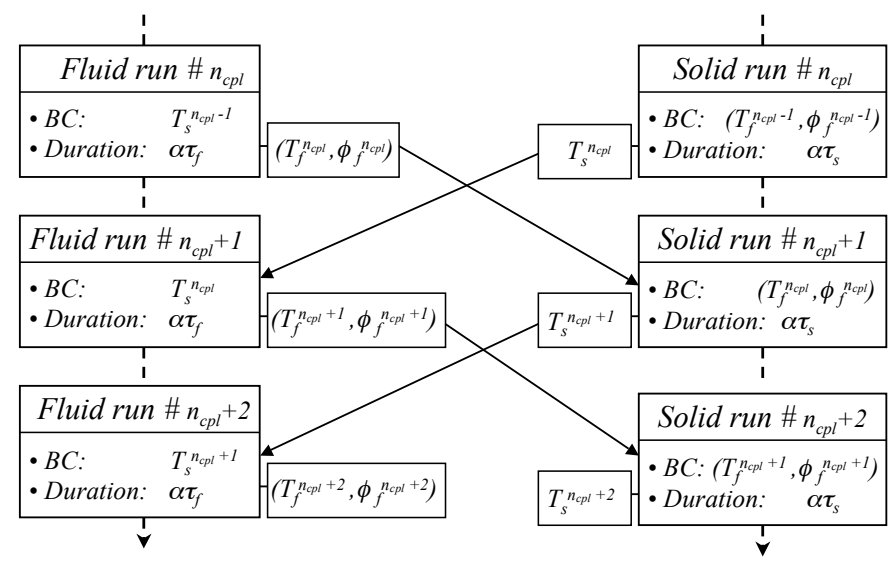

Figure 2: Parallel coupling strategy PCS. 


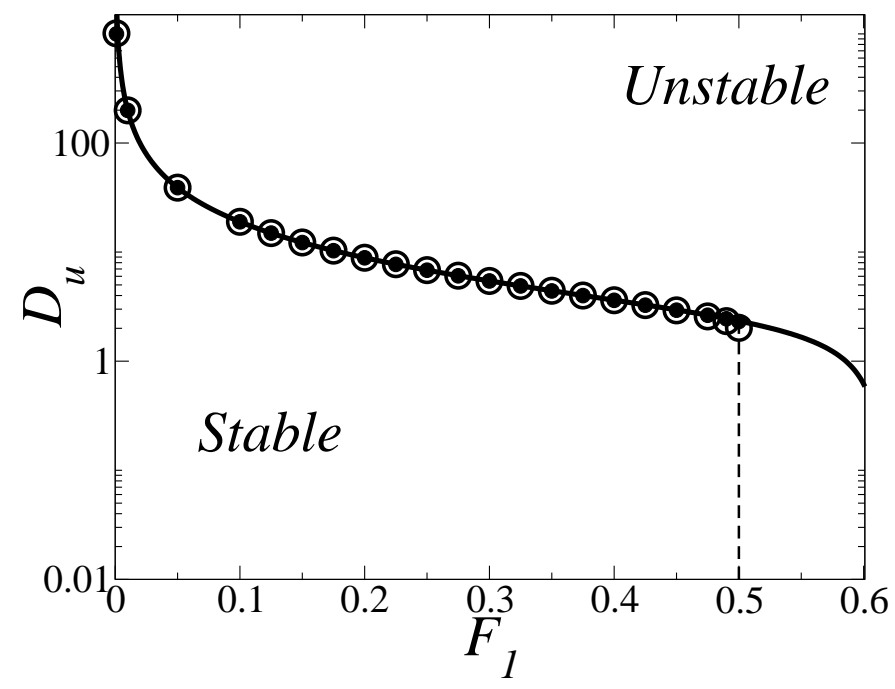

Figure 3: Upper limit of stability $\mathcal{D}_{u}$ for the discretisation Eq. 22 as a function of Fourier Number $\mathcal{F}_{1}$ : -, analytical solution for $N_{1}=4 ; \bullet$, computed numerically with $N_{1}=4 ; \circ$, computed numerically with $N_{1}=51$. 


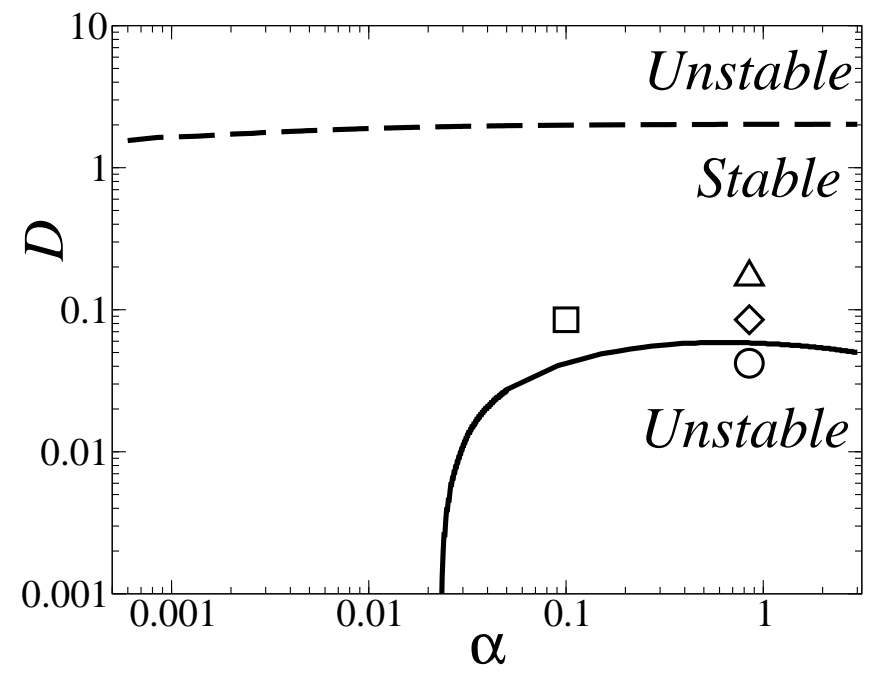

Figure 4: Stability map in the $(\alpha, \mathcal{D})$ space of the simplified conjugate heat transfer problem $\mathcal{P}_{\mathcal{S C H}}$ (Eq. 16 and 18): -, lower stability limit $\mathcal{D}_{m} ;--$ upper stability limit $\mathcal{D}_{M} . \alpha$ is the coupling synchronization time parameter (Eq. 27) and $\mathcal{D}$ is a function of the coupling relaxation parameter $h$ (Eq. 23). Positions of some computations done on T120D blade (Section 3): point $\left(\alpha_{1}, h_{2}\right) \square$; point $\left(\alpha_{2}, h_{1}\right) \bigcirc$; point $\left(\alpha_{2}, h_{2}\right) \diamond$ and point $\left(\alpha_{2}, h_{3}\right) \triangle$. The parameters $\left(\alpha_{i}, h_{j}\right)$ are given in Tab. 6 . 


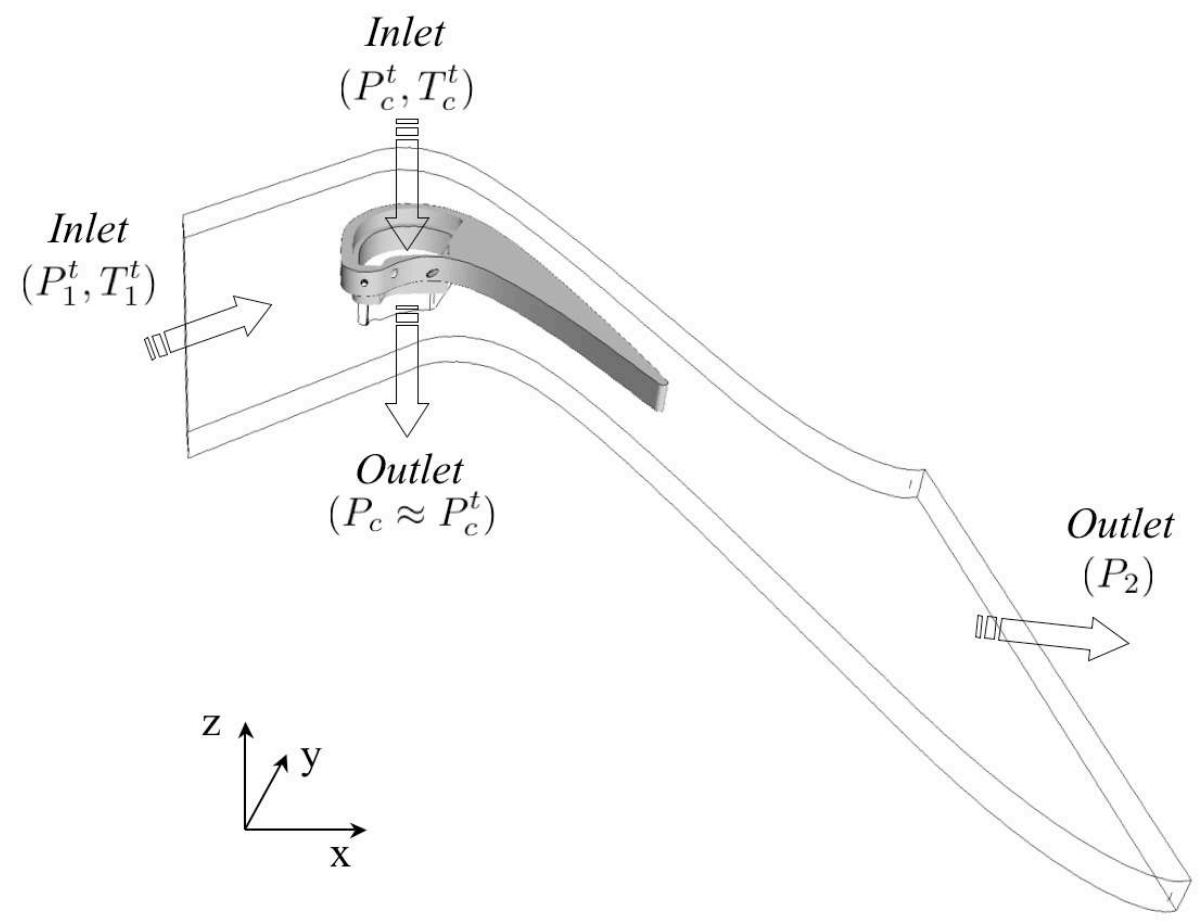

Figure 5: Fluid computational domain. 


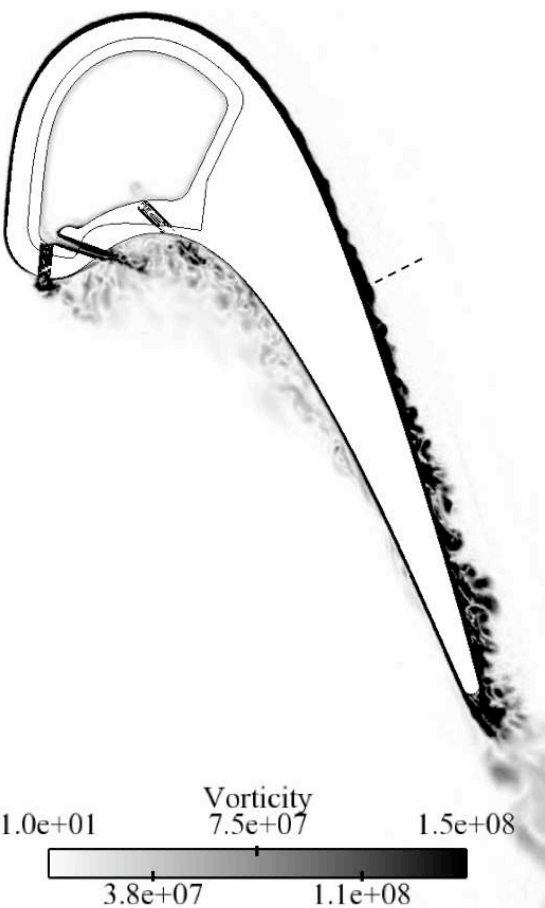

(a)

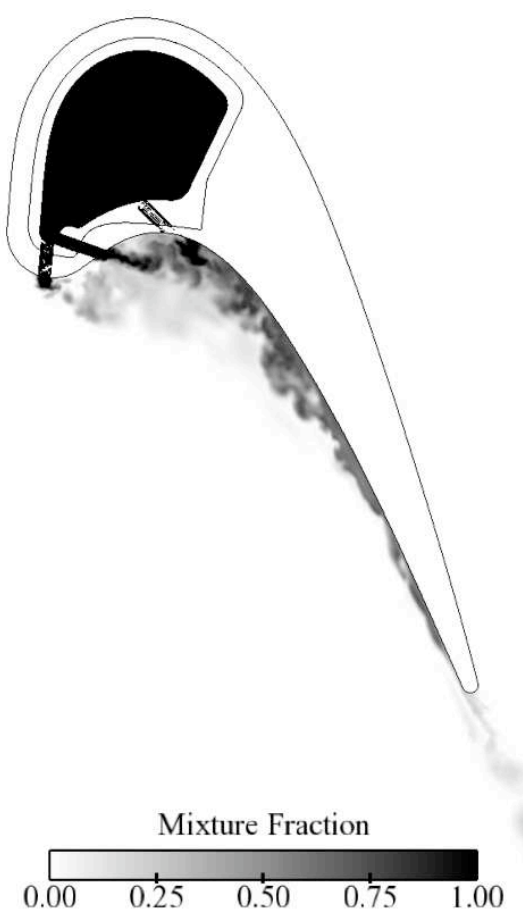

(b)

Figure 6: Instantaneous snapshot of (a) vorticity and (b) distribution of cooling air within a cutting plane at constant $z$ passing thought jet 2 . The dashed line on (a) represents the approximate position of the shock at $75 \%$ of the axial chord. 


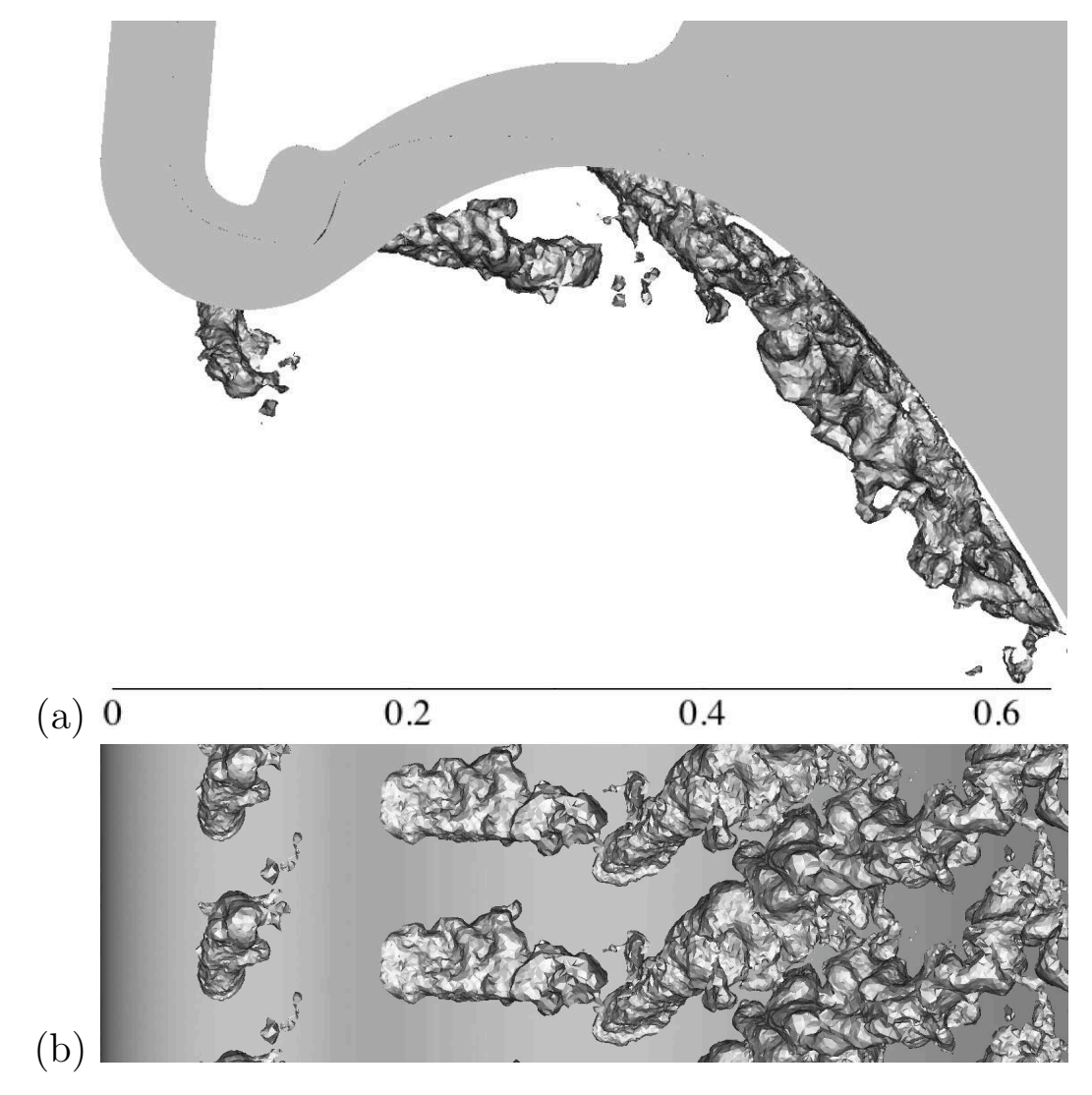

Figure 7: Instantaneous isosurface of temperature $T=318 K$ : (a) $z$ view and (b) $y$ view. 


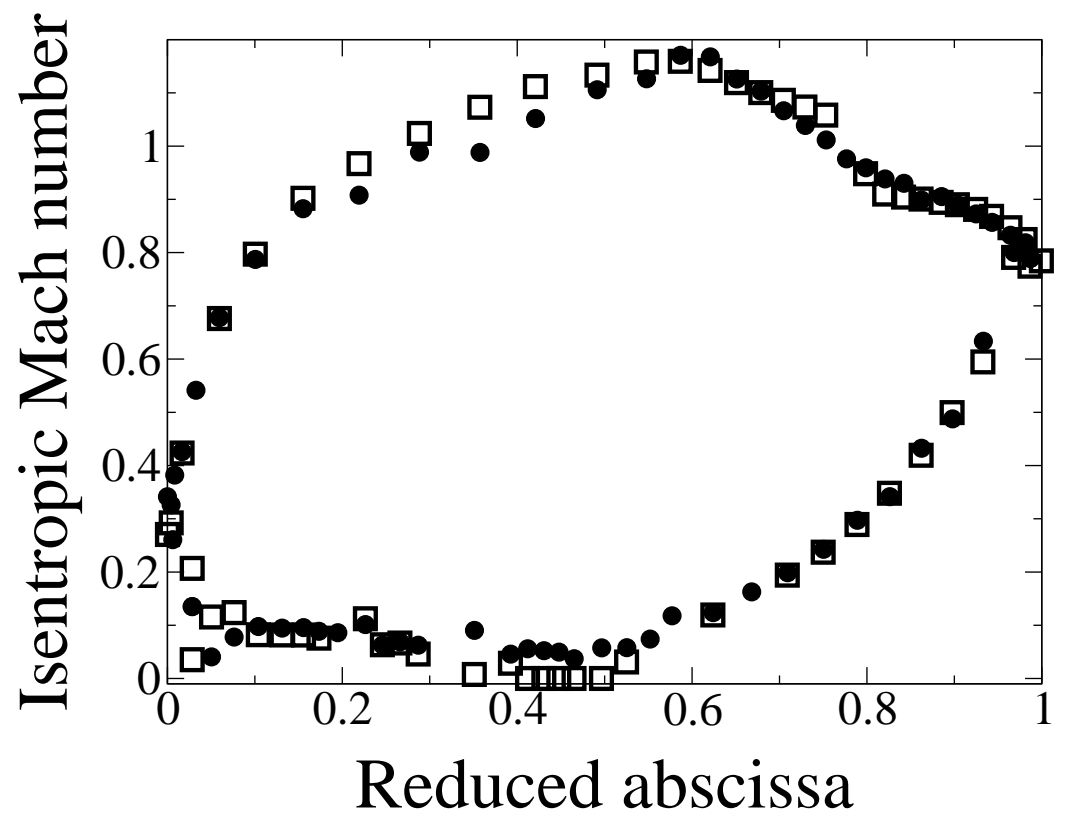

Figure 8: Time-averaged isentropic Mach number (Eq. 29) along the blade at pressure probe locations as a function of reduced abscissa: •, adiabatic LES; $\square$, experiment. 


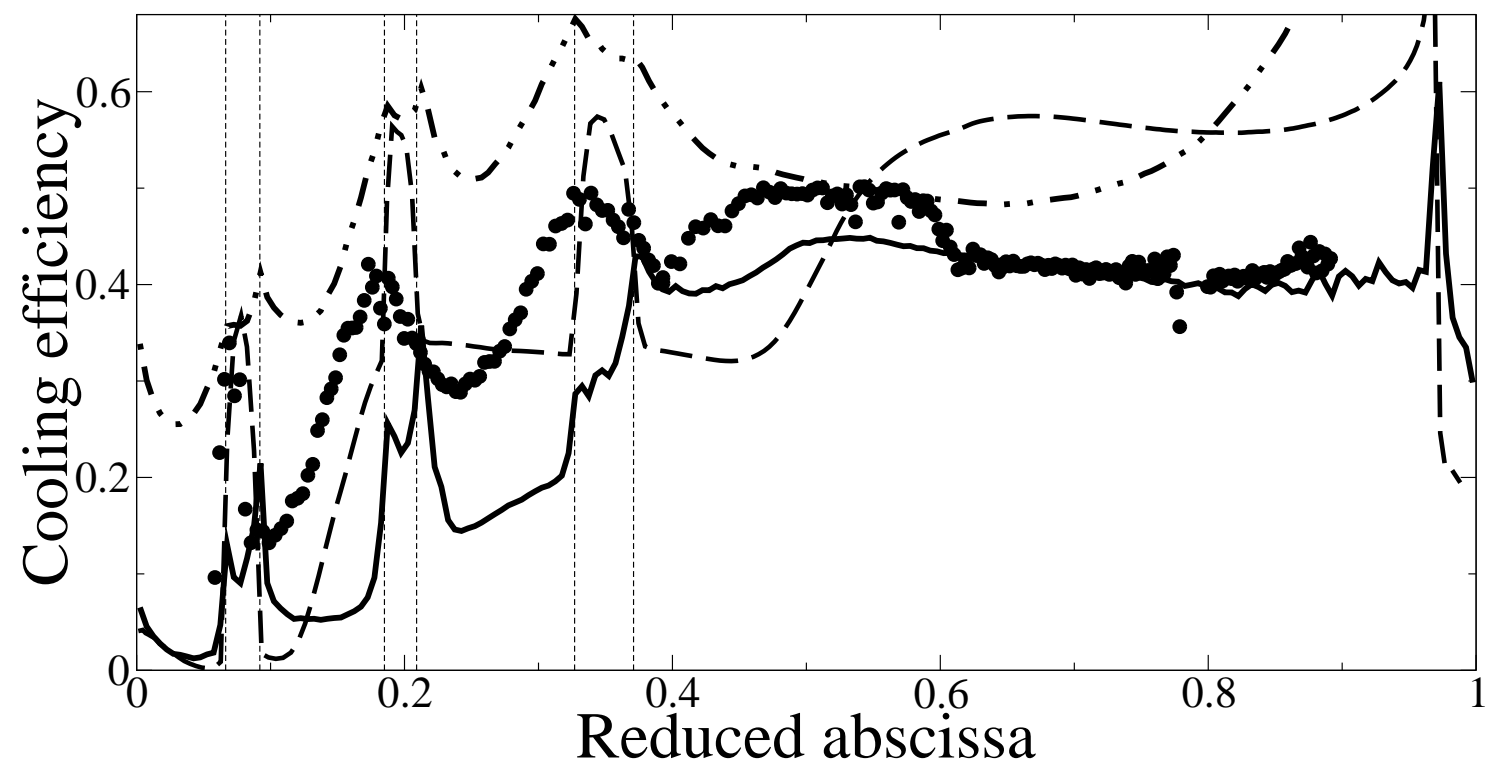

Figure 9: Time and spanwise averaged cooling efficiency (Eq. 31) versus abscissa on the pressure side as a function reduced abscissa: •, experiment from UNIBW; - adiabatic LES; -- adiabatic RANS; - - - coupled LES. 


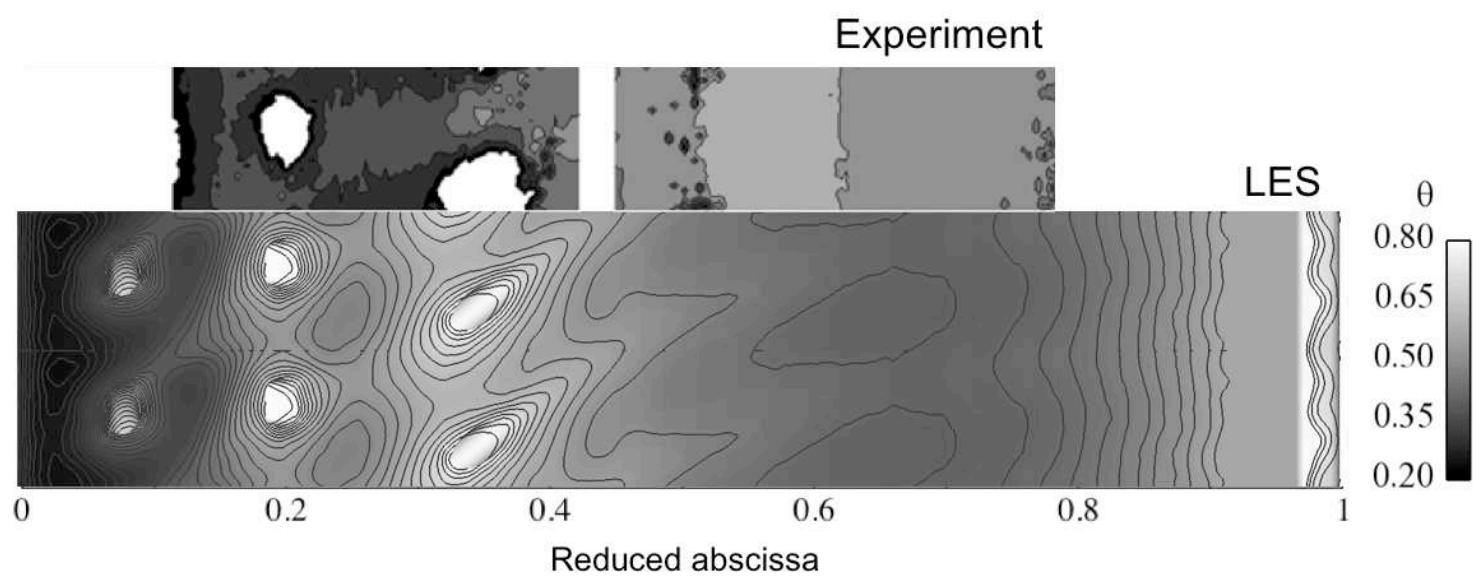

Figure 10: 2D plot of time averaged cooling efficiency on the pressure side: comparison of experimental results and coupled simulation. The scale of $\Theta$ corresponds only to the LES field. 


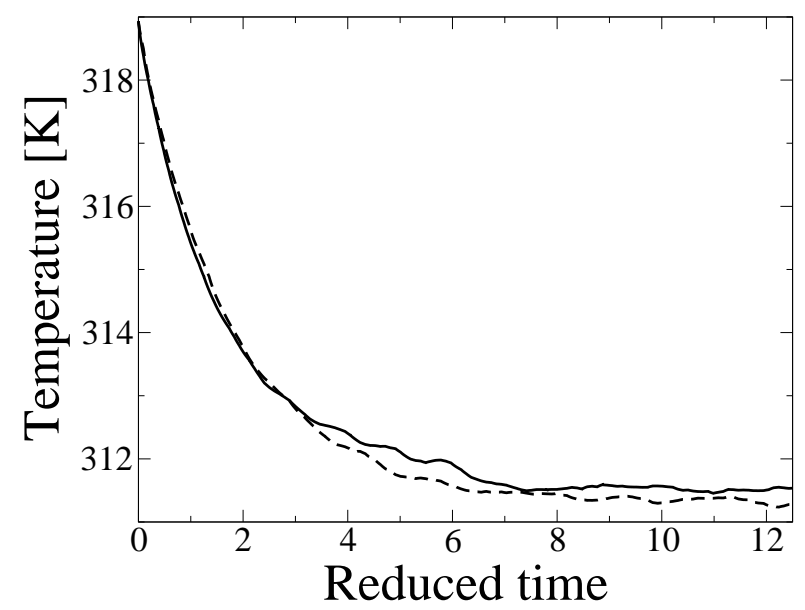

Figure 11: Evolutions of the mean temperature in the blade as a function of reduced solid time $t_{s} / \tau_{s}$ for $S C S\left(\alpha_{1}, h_{2}\right)$ - and $P C S\left(\alpha_{1}, h_{2}\right)--$. The parameters $\left(\alpha_{1}, h_{2}\right)$ are given in Tab. 6. As a reference for restitution times, one solid characteristic time $\tau_{s}$ requires almost a total of 295 CPU hours for the LES and the heat conduction solvers on a SGI Altix ICE8200. 


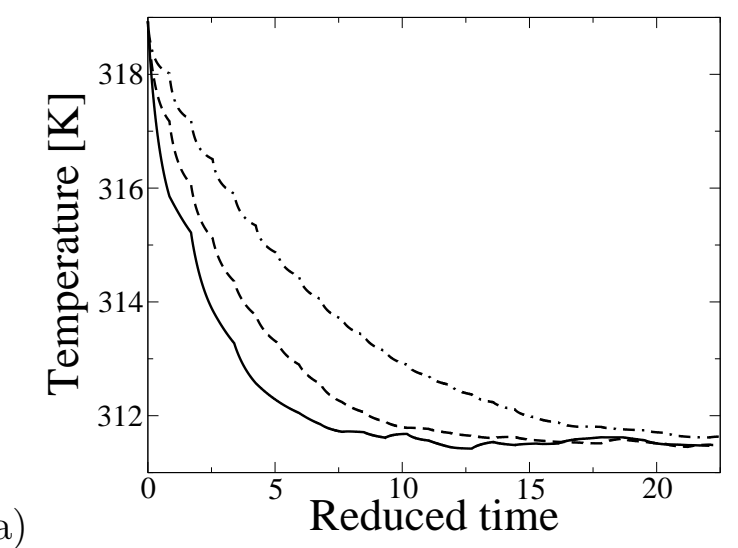

(b)

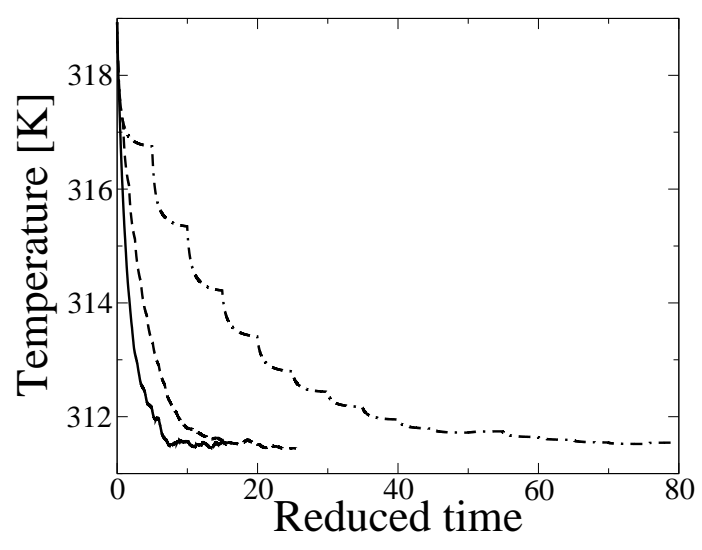

Figure 12: Evolutions of the mean temperature in the blade as a function of reduced solid time $t_{s} / \tau_{s}$ for (a) $S C S\left(\alpha_{2}, h_{1}\right)-, S C S\left(\alpha_{2}, h_{2}\right)--$ and $S C S\left(\alpha_{2}, h_{3}\right)-\cdot-$; and for (b) $S C S\left(\alpha_{1}, h_{2}\right)-, S C S\left(\alpha_{2}, h_{2}\right)--$ and $S C S\left(\alpha_{3}, h_{2}\right)-\cdot-$. The parameters $\left(\alpha_{i}, h_{j}\right)$ are given in Tab. 6. As a reference for restitution times, one solid characteristic time $\tau_{s}$ requires almost a total of $295 \mathrm{CPU}$ hours for the LES and the heat conduction solvers on a SGI Altix ICE8200. 